\title{
Crossover scaling in two dimensions
}

\author{
Erik Luijten* and Henk W. J. Blöte \\ Department of Physics, Delft University of Technology, Lorentzweg 1, 2628 CJ Delft, The Netherlands \\ Kurt Binder \\ Institut für Physik, WA 331, Johannes Gutenberg-Universität, D-55099 Mainz, Germany
}

(Received 4 June 1997)

\begin{abstract}
We determine the scaling functions describing the crossover from Ising-like critical behavior to classical critical behavior in two-dimensional systems with a variable interaction range. Since this crossover spans several decades in the reduced temperature as well as in the finite-size crossover variable, it has up to now largely evaded a satisfactory numerical determination. Using a dedicated Monte Carlo method, we could obtain accurate results for sufficiently large interaction ranges. Our data cover the full crossover region both above and below the critical temperature and support the hypothesis that the crossover functions are universal. Also the so-called effective exponents are discussed and we show that these can vary nonmonotonically in the crossover region. [S1063-651X(97)02512-9]
\end{abstract}

PACS number(s): 64.60.Fr, 75.40.Cx, 75.10.Hk, 05.70.Fh

\section{INTRODUCTION}

The crossover from Ising-like to classical critical behavior has attracted renewed attention in recent years. This crossover behavior occurs in many thermodynamic systems, such as ionic solutions, simple fluids, fluid mixtures, and polymer mixtures. The Ginzburg criterion [1] states that sufficiently close to the critical point these systems exhibit critical exponents belonging to the three-dimensional (3D) Ising universality class. At larger distances from the critical point, but still within the critical region, classical (mean-field-like) critical exponents are observed. Although this appears to be a well-established picture, the precise nature of the crossover between these two universality classes is still subject to investigation. For example, Anisimov et al. recently claimed [2] to have observed an "effective" susceptibility exponent that varied nonmonotonically from its classical value $\gamma_{\mathrm{MF}}$ $=1$ to its Ising value $\gamma_{I} \approx 1.24$ when the critical point was approached. Later, the possibility of such behavior within the critical domain was questioned by Bagnuls and Bervillier; see Refs. [3,4]. On the other hand, Fisher has argued [5] that nonmonotonic variation of effective critical exponents is not necessarily an indication of nonuniversal behavior. Other questions concern the size of the crossover region, which is expected to span several decades in the crossover variable [6], and the size of the temperature region around $T_{c}$, within which Ising-like behavior is observed [7]. Until now it has turned out to be very difficult to accurately observe the full crossover region in numerical simulations. A major effort has been undertaken in Ref. [8] for three-dimensional polymer mixtures, where crossover occurs as a function of the polymer chain length. However, despite chain lengths of up to 512 monomers, the results did not span the full crossover region. For this reason, Mon and Binder [9] turned their attention to the two-dimensional Ising model with an extended range of interaction, where a crossover from Ising-

*Electronic address: erik@tntnhb3.tn.tudelft.nl like to classical critical behavior occurs when the range $R$ of the spin-spin interactions is increased (suppressing the critical fluctuations). In two dimensions not only one can access larger interaction ranges, but also both asymptotic regimes are known exactly and the variation of the critical exponents is considerably larger than in the crossover from 3D Isinglike critical behavior to classical critical behavior. Mon and Binder derived the (singular) $R$ dependence of the critical amplitudes of scaling functions and carried out Monte Carlo simulations to verify these predictions numerically. Even in these two-dimensional systems, the mean-field regime turned out to be only barely reachable.

In a recent paper [10] we rederived the predictions of Mon and Binder from renormalization theory and also obtained the $R$ dependence of various corrections to scaling, such as the shift of the critical temperature with respect to the mean-field critical temperature. Furthermore, larger interaction ranges and system sizes were accessible to our numerical simulations thanks to a dedicated Monte Carlo algorithm. This enabled us to actually verify the theoretical predictions in two-dimensional systems. In this paper we show that the simulations presented in Ref. [10] allow a full mapping of the finite-size crossover curves for various quantities. However, these curves describe the finite-size dependences of critical amplitudes, which (to our knowledge) have not been observed experimentally. Therefore, we have also carried out simulations at temperatures farther from the critical temperature in order to observe the thermal crossover of these quantities. The results of these simulations, which partially have been reported in an earlier paper [11], are presented as well. The fact that in our model both the temperature distance from the critical point and the interaction range can be varied turns out to be essential to observe the full crossover region.

The outline of the remainder this paper is as follows. After a short recapitulation of the model under investigation (Sec. II) we start in Sec. III with finite-size crossover scaling. We discuss the required system sizes and interaction ranges and obtain the crossover curves for the absolute magnetiza- 
tion density, magnetic susceptibility, the spin-spin correlation function over half the system size, and the fourth-order amplitude ratio. Thermal crossover scaling is treated in Sec. IV, where we consider the approach of $T_{c}$ both in the symmetric phase $\left(T>T_{c}\right)$ and in the state of broken symmetry $\left(T<T_{c}\right)$. Again, crossover curves are obtained for the order parameter and the susceptibility. The various aspects of these curves are discussed in some detail. Graphs of the logarithmic derivatives of the crossover curves, which can be associated with so-called effective critical exponents as measured in experiments, are presented in Sec. V. In Sec. VI we end with a summary of our conclusions.

\section{SHORT DESCRIPTION OF THE MODEL}

Let us first briefly recall the model as it was introduced in Ref. [9]. This is a two-dimensional Ising system consisting of $L \times L$ lattice sites with periodic boundary conditions. Each spin in the system interacts equally with its $z$ neighbors lying within a distance $R_{m}$. This defines the coupling between two spins $s_{i}$ and $s_{j}$ at a distance $r$ as

$$
K_{i j}=K(r) \equiv \begin{cases}c R_{m}^{-d} & \text { if } r \leqslant R_{m} \\ 0 & \text { if } r>R_{m} .\end{cases}
$$

In the absence of an external magnetic field the Hamiltonian is

$$
\mathcal{H} / k_{B} T=-\sum_{i} \sum_{j>i} K\left(\left|\mathbf{r}_{i}-\mathbf{r}_{j}\right|\right) s_{i} s_{j},
$$

where the sums run over all spins in the system and $\mathbf{r}_{i}$ denotes the position of spin $s_{i}$. To suppress lattice effects we use an effective interaction range $R$, defined as

$$
R^{2} \equiv \frac{\sum_{j \neq i}\left(\mathbf{r}_{i}-\mathbf{r}_{j}\right)^{2} K_{i j}}{\sum_{j \neq i} K_{i j}}=\frac{1}{z} \sum_{j \neq i}\left|\mathbf{r}_{i}-\mathbf{r}_{j}\right|^{2} \quad \text { with }\left|\mathbf{r}_{i}-\mathbf{r}_{j}\right| \leqslant R_{m} .
$$

For large ranges, $R$ approaches the limiting value $R_{m} / \sqrt{2}$.

\section{FINITE-SIZE CROSSOVER SCALING}

\section{A. General considerations}

It has been shown by Binder and Deutsch [12] that crossover scaling can be combined with finite-size scaling by including the dependence on the crossover variable in the probability distribution function of the order parameter. Indeed, just as crossover in the thermodynamic limit is described as a function of the reduced temperature divided by the Ginzburg number, it can be described as the function of a size-dependent crossover variable $G$ in finite systems. In Ref. [9] this crossover variable was derived as $G$ $=L R^{-4 /(4-d)}$, where $L$ is the linear system size and $d$ denotes the dimensionality. This also follows from the renormalization treatment in Ref. [10]. In short, sufficiently close to the Gaussian fixed point (i.e., for a sufficiently large interaction range $R$ ) the critical behavior will be classical. In terms of a renormalized Landau-Ginzburg-Wilson (LGW)
Hamiltonian in momentum space, this implies that the coefficient of the $\phi^{4}$ term must be much smaller than that of the $\phi^{2}$ term, $u L^{4-d} / R^{4} \ll 1$ [cf. in particular Eq. (6) of Ref. [10]], which again leads to the crossover parameter $L R^{-4 /(4-d)}$, where for the moment we assume that $u$ is of order unity.

In Ref. [10] we focused our attention on the critical finitesize amplitudes in the limit of $L \rightarrow \infty$. Here we will examine the crossover in the corresponding data for finite system sizes. Since the crossover regime is expected $[5,6]$ to span several decades in the crossover variable $G=L / R^{2}$, it is numerically not feasible to observe both asymptotic regimes by merely varying the system size $L$ while keeping the range $R$ fixed. Therefore, we construct the curve by combining the results for various values of $R$; cf. Ref. [8]. Indeed, the Ising regime $\left(L / R^{2} \gg 1\right)$ is easily reachable, although the results for very small ranges do not conform well to the leading $R$ dependence of the critical scaling functions [9] and are thus, at first sight, not well suited for constructing the crossover curve. The mean-field regime $\left(L / R^{2} \ll 1\right)$, however, poses more substantial problems. If the linear system size $L$ is made too small, the numerical results exhibit strong finitesize effects. Therefore, $L$ must be at least of the order of the interaction range. More precisely, boundary effects will occur for systems for which $L \approx R_{m}$ and the smallest possible value of the crossover variable $G$ is roughly equal to $R_{m} / R^{2} \approx \sqrt{2} / R$. Thus large ranges are required to reach the regime where $G \ll 1$. In a conventional Monte Carlo algorithm, the efficiency of simulations rapidly decreases with increasing interaction range. This limitation has been circumvented by applying a dedicated cluster algorithm, as explained in Ref. [10]. Still, a problem remains. Namely, the finite-size crossover scaling is valid $a t$ the critical temperature. Any deviation from this temperature will lead to systematic errors in the analysis. Since the (range-dependent) critical temperatures are determined in the Ising limit, i.e., from system sizes $L>R^{2}$, large interaction ranges require very large system sizes for an accurate determination of $T_{c}$. For example, the most efficient way to obtain data for $G$ $\approx 0.02$ is to simulate a system with $L=100$ and $R_{m}=100$ $(R \approx 70)$. However, an accurate determination of $T_{c}(R$ $=70$ ) requires system sizes of at least $L=5000$, whereas we have carried out simulations for system sizes up to 1000 $\times 1000$ lattice sites. This has been solved as follows. The renormalization treatment in Ref. [10] predicts the form of the function describing how $T_{c}(R)$ deviates from the meanfield critical temperature when $R$ varies. By fitting this function to the accurately determined critical temperatures in our previous study an expression is obtained for $T_{c}(R)$ from which the critical temperatures for very large ranges can be calculated to a relatively high accuracy. The shift of $T_{c}$ is expressed by

$$
T_{c}=T_{c}^{\mathrm{MF}}+\frac{a_{1}}{R^{2}}\left[1+a_{2} \ln R^{2}\right]+\frac{a_{3}}{R^{4}},
$$

where $T_{c}^{\mathrm{MF}}=1$ and the last term is a higher-order correction omitted in Ref. [10]. A least-squares fit for $16 \leq R^{2} \leq 70$ $\left(32 \leqslant R_{m}^{2} \leqslant 140\right.$ in Ref. [10]) yielded $a_{1}=-0.267(6), a_{2}$ $=1.14(3)$, and $a_{3}=-0.27(3)$. Figure 1 shows the critical temperatures and expression (4) with the appropriate coefficients. 

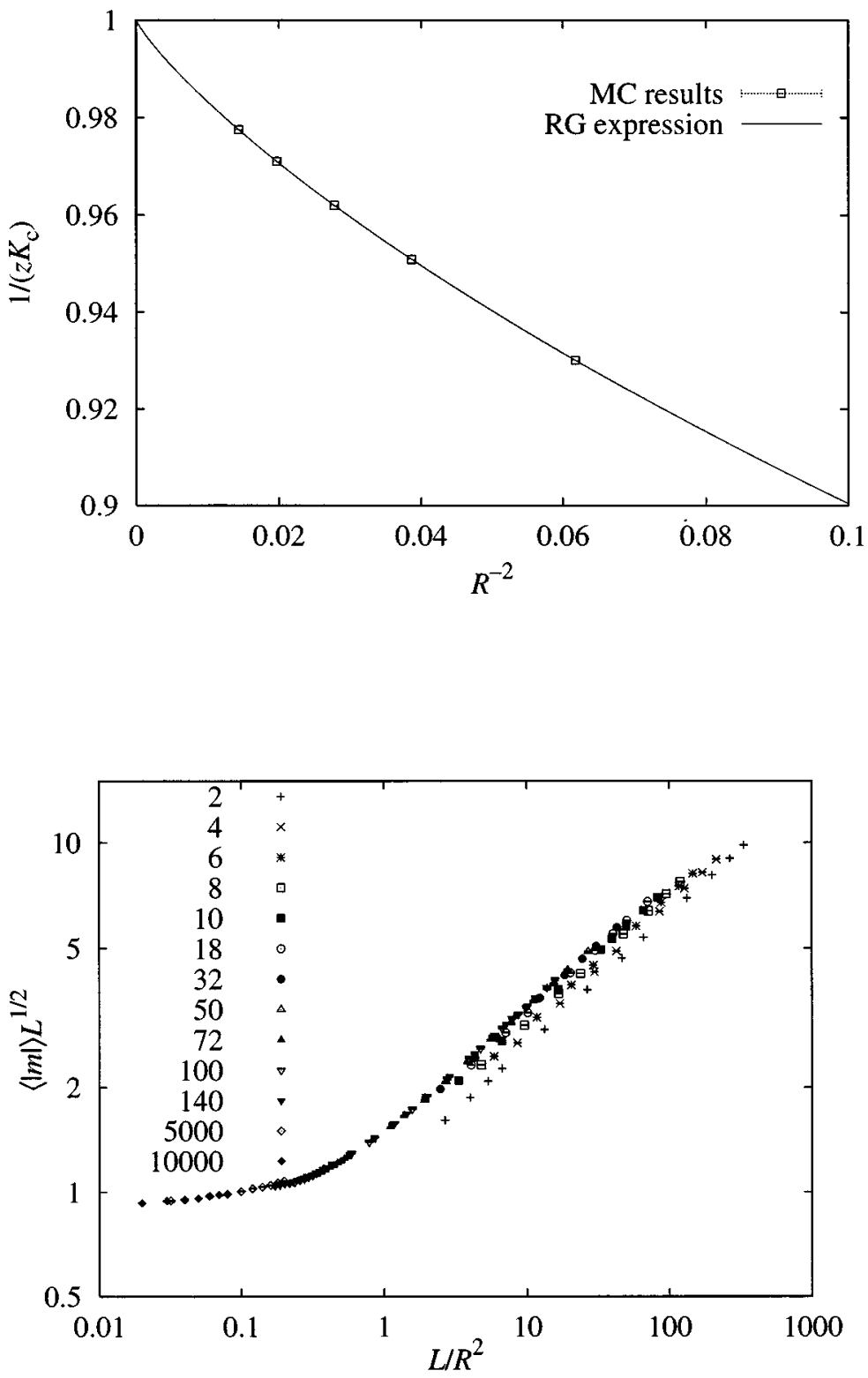

(a)

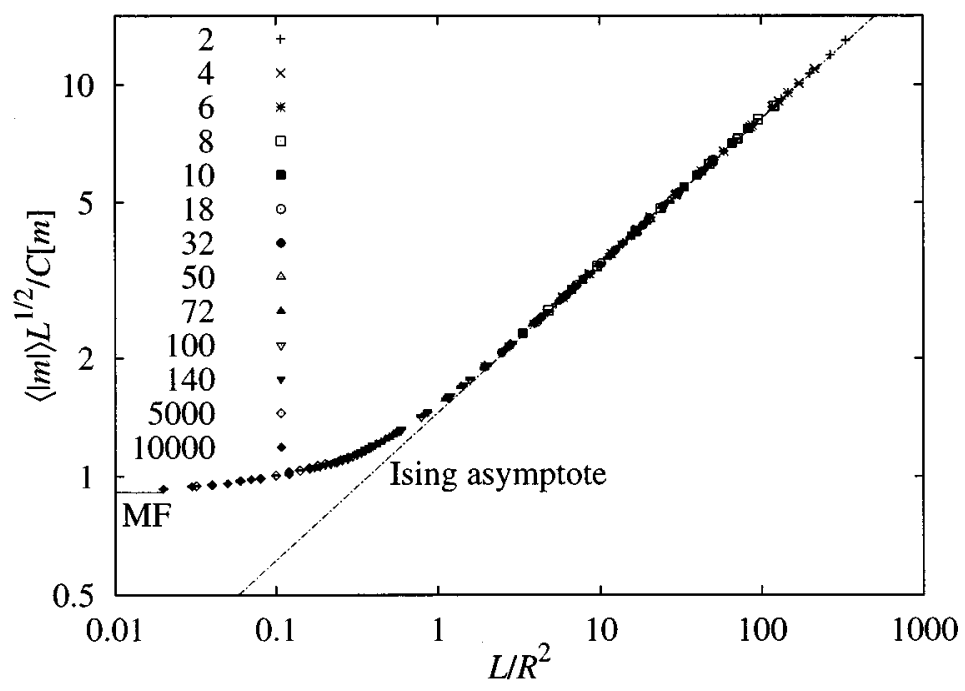

FIG. 1. Critical temperature as a function of the inverse interaction range, together with the renormalization expression (4) fitted to it.
FIG. 2. (a) Finite-size crossover curve for the absolute magnetization density multiplied by the square root of the system size. (b) Same graph, but now the range-dependent corrections predicted by renormalization theory have been divided out. The correction factor abbreviated by $C[m]$ stands for the factor in square brackets in Eq. (6). A perfect collapse is obtained for all system sizes and interaction ranges. Both the exact mean-field limit (indicated by "MF") and the Ising asymptote with slope $3 / 8$ are confirmed by the data. In this and all following figures the numbers in the key refer to values for $R_{m}^{2}$. 


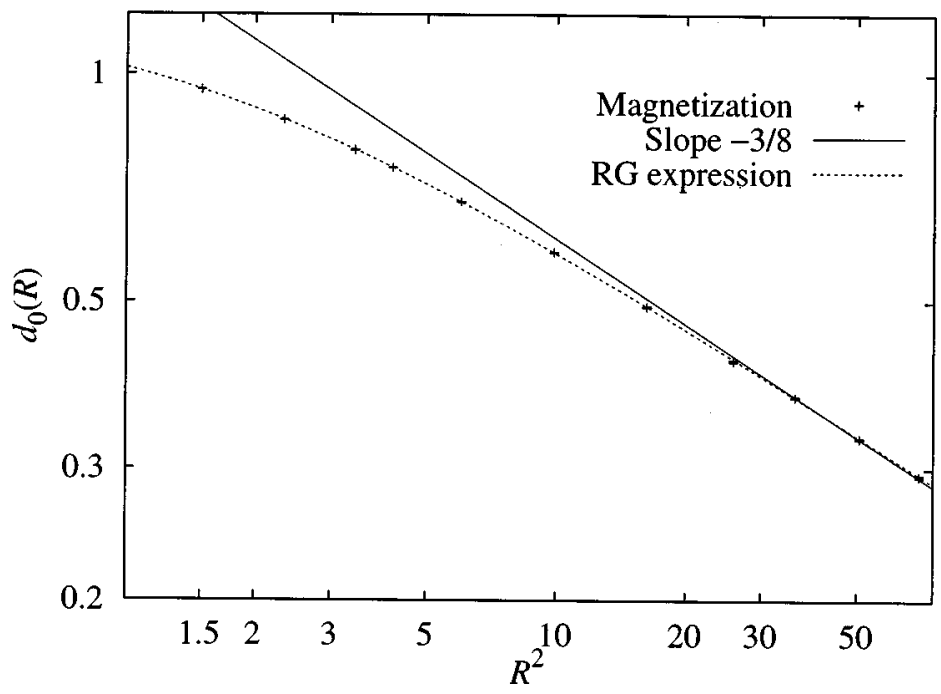

\section{B. Absolute magnetization density}

In the Ising regime, the absolute magnetization density scales (at criticality) asymptotically as $\langle|m|\rangle=L^{-1 / 8} d_{0}(R)$, where the critical amplitude $d_{0}$ is a function of $R, d_{0}$ $\propto R^{-3 / 4}$. In the mean-field regime $\langle|m|\rangle$ does not depend on $R$, but is simply proportional to $L^{-1 / 2}$. When plotting $\langle|m|\rangle$ as a function of $G=L / R^{2}$ a data collapse is obtained if it is multiplied by a factor $L^{x} R^{-(2 x-1)}$. This resulting quantity is proportional to $G^{x-1 / 8}$ in the Ising regime and to $G^{x-1 / 2}$ in the mean-field regime. A suitable choice is $x=1 / 2$ because this yields a quantity that is still independent of $R$ in the mean-field regime. Indeed, it is shown in the Appendix that in a two-dimensional system in which all spin-spin interactions are equally strong

$$
\langle|m|\rangle=12^{1 / 4} \frac{\Gamma\left(\frac{1}{2}\right)}{\Gamma\left(\frac{1}{4}\right)} \frac{1}{\sqrt{L}}+O\left(\frac{1}{L^{3 / 2}}\right)
$$

and $\langle|m|\rangle \sqrt{L}$ will thus approach $12^{1 / 4} \Gamma(1 / 2) / \Gamma(1 / 4)$ $=0.909890588 \ldots$ in the limit of $G \rightarrow 0$. We remark that our requirement $L>\sqrt{2} R$ unambiguously relates the limit $G \rightarrow 0$ to the mean-field $(R \rightarrow \infty)$ limit. In Fig. 2(a) we have plotted the absolute magnetization density multiplied by the square root of the system size versus the crossover variable. Interaction ranges from $R_{m}^{2}=2$ to $R_{m}^{2}=10000$ were included, where the data for $R_{m}^{2}=5000$ and $R_{m}^{2}=10000$ (spanning the range $0.02 \lesssim G \leqq 0.2$ ) have been obtained at temperatures calculated from Eq. (4): $K_{c}\left(R_{m}=\sqrt{5000}\right)$ $=6.3746(3) \times 10^{-5}$ and $K_{c}\left(R_{m}=\sqrt{10000}\right)=3.18491(9)$ $\times 10^{-5}$. The crossover curve evidently spans approximately three decades in $G$. In the limit of $G \rightarrow 0$ it gradually approaches a horizontal line. For $G \gg 1$ the picture is not very clear. The data points for each single value of $R$ lie on a straight line with slope $3 / 8$, corresponding to the Ising asymptote, but the asymptotes only coincide for large ranges (cf. Fig. 4 in Ref. [9]). The reason for this is that, as mentioned above, for small ranges the critical amplitudes do not conform to the leading $R^{-3 / 4}$ dependence. This can be cured by invoking the renormalization treatment of Ref. [10]. Indeed, the theory predicts the structure of the corrections to the leading $R$ dependence of the critical amplitude,
FIG. 3. Critical amplitude of $\langle|m|\rangle$ and the renormalization prediction fitted to it. This correction factor is used in Fig. 2(b).

$$
d_{0}=b_{0} R^{-3 / 4}\left[1+\frac{1}{R^{2}}\left(b_{1}+b_{2} \ln R^{2}\right)\right]
$$

This "finite-range correction" is very similar to the shift of the critical temperature in Eq. (4), but originates from a different term in the renormalized LGW Hamiltonian. To illustrate this correction graphically, we have reproduced Fig. 6 from Ref. [10] and included the result of a least-squares fit of Eq. (6) to the data; see Fig. 3. The curve clearly yields an excellent description of the critical amplitudes, even for small ranges. We have used this fit to construct a clear crossover curve for the magnetization density on which the data for all values of $R$ collapse. To this end, all data are divided by the correction factor in square brackets in Eq. (6). The result is shown in Fig. 2(b). One observes that in the Ising regime all data perfectly collapse on a common asymptote with slope 3/8. For $G$ small, the data indeed approach the mean-field prediction (5). The fact that at $G \approx 0.2$ the data for $R_{m}^{2}=5000$ and $R_{m}^{2}=10000$ coincide with those for $R_{m}^{2}$ $=72,100,140$ confirms that the critical temperatures for the large ranges have been estimated accurately. The center of the crossover region lies between $G=0.1$ and $G=1.0$, which shows that the parameter $u$ is indeed of order unity. Finally, it is particularly encouraging that no remaining finite-size effects, causing deviations from the curve, are visible in Fig. 2(b), despite the fact that the correction factor was calculated in the $L \rightarrow \infty$ limit and hence does not compensate for such higher-order finite-size effects.

\section{Magnetic susceptibility}

The procedure described above for the absolute magnetization density can be applied to the magnetic susceptibility $\chi$, which we have calculated from the average square magnetization; $\chi=L^{d}\left\langle m^{2}\right\rangle$. At $T=T_{c}$, the susceptibility is in the Ising regime proportional to $L^{7 / 4} R^{-3 / 2}$ and in the mean-field regime it scales proportionally to $L$. To obtain a data collapse for $\chi$ as a function of $G$, one has to multiply the finite-size data by $L^{x} R^{-(2 x+2)}$, where a suitable choice is given by $x$ $=-1$. In the mean-field limit, $\chi / L$ approaches $\sqrt{12} \Gamma(3 / 4) / \Gamma(1 / 4)=1.17082866 \ldots$ (see the Appendix). As shown in Ref. [10], the deviation from the leading range 


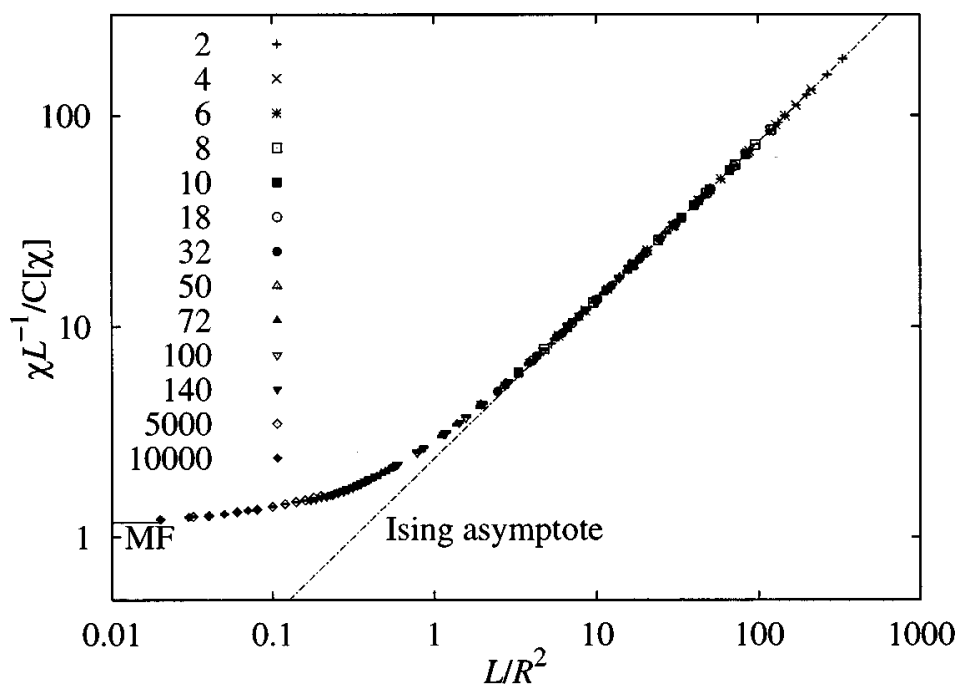

FIG. 4. Finite-size crossover curve for the magnetic susceptibility divided by the system size. The range-dependent correction factor $C[\chi]$ [the factor in square brackets in Eq. (7)] has been divided out, as discussed in the text. Both the mean-field limit and the Ising asymptote (slope 3/4) are confirmed by the data. dependence of the critical amplitude is very similar to that of the absolute magnetization density,

$$
p_{0}=q_{0} R^{-3 / 2}\left[1+\frac{1}{R^{2}}\left(q_{1}+q_{2} \ln R^{2}\right)+\frac{q_{3}}{R^{4}}\right],
$$

where now one additional higher-order correction is required. Therefore, we only show the resulting crossover curve for the susceptibility after the data have been divided by the correction factor in square brackets; see Fig. 4. Again, both the mean-field asymptotic result and the Ising asymptote (slope 3/4) are clearly reproduced, with a perfect collapse for all ranges.

\section{Spin-spin correlation function}

Closely related to the magnetic susceptibility is the spinspin correlation function $g(|\mathbf{r}|)$. In our simulations we have sampled $g(L / 2)$, which scales both in the Ising regime and in the mean-field regime as $\chi / L^{2}$. Thus we obtain a data collapse by multiplying the finite-size data by $L^{x} R^{-(2 x-2)}$, in which we have set $x=1$. After correcting for the higherorder range-dependent corrections in the critical amplitude [which have the same structure as those in Eq. (7)] we obtain the graph shown in Fig. 5. The full crossover curve can be mapped and shows a close resemblance to that for the susceptibility, including the approach of the asymptotic meanfield value. In the range $0.2 \lesssim L / R^{2} \leqslant 1.0$, the data do not precisely coincide on a smooth curve. This is due to nonlinear finite-size effects, which are, for the spin-spin correlation function, apparently larger than for the absolute magnetization density or the magnetic susceptibility. We will pay more attention to these deviations when discussing the universal amplitude ratio (see below). It should be noted that the critical amplitudes listed in Table V of Ref. [10] have to be multiplied by a factor $2^{-1 / 4}=0.84089642 \ldots$ in order to obtain the correct values.

\section{E. Universal amplitude ratio}

The amplitude ratio $Q_{L} \equiv\left\langle m_{L}^{2}\right\rangle^{2} /\left\langle m_{L}^{4}\right\rangle$ is a size-dependent quantity, which takes a universal value $Q$ in the $L \rightarrow \infty$ limit. That is, it is calculated by taking the ratio of the square of the magnetization density and the fourth power of it in a finite geometry and subsequently taking the limit $L \rightarrow \infty$. For $T$ $>T_{c}, Q$ approaches the Gaussian value $Q=1 / 3$ and for $T$ $<T_{c}$ it approaches the maximum value $Q=1$. At criticality, the amplitude ratio is known exactly in the mean-field case, $Q_{\mathrm{MF}}=0.45694658 \ldots[13,14]$ and to a high accuracy in the two-dimensional Ising model; $Q_{\mathrm{I}} \approx 0.856216(1)$ [15]. In

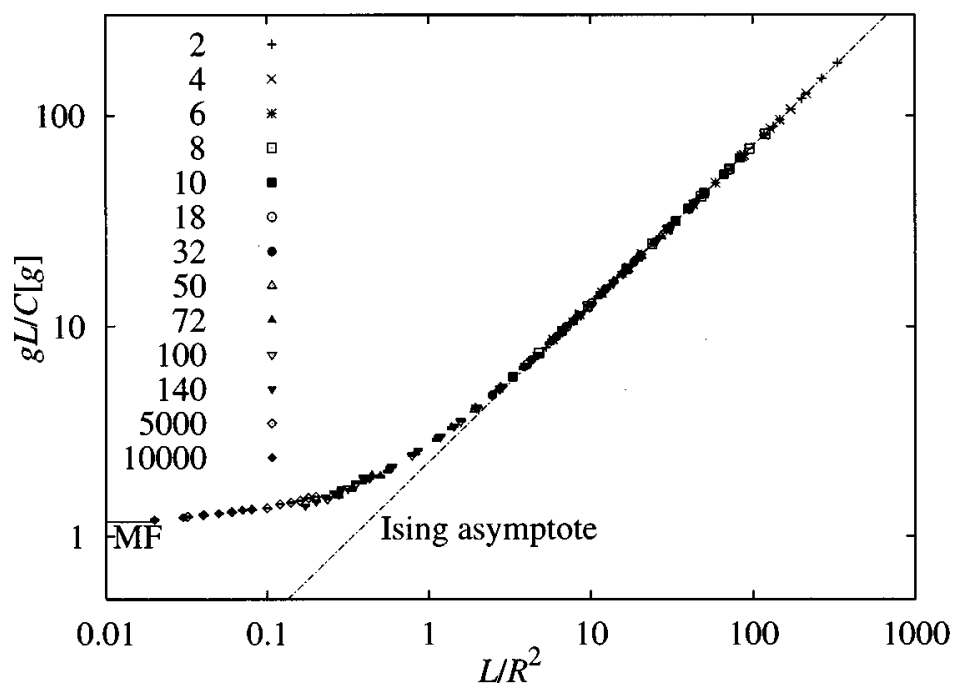

FIG. 5. Finite-size crossover curve for the spin-spin correlation function multiplied by the system size. A range-dependent correction factor (abbreviated as $C[g]$ ) has been divided out, as discussed in the text. Both the mean-field limit and the Ising asymptote (slope 3/4) are confirmed by the data. 


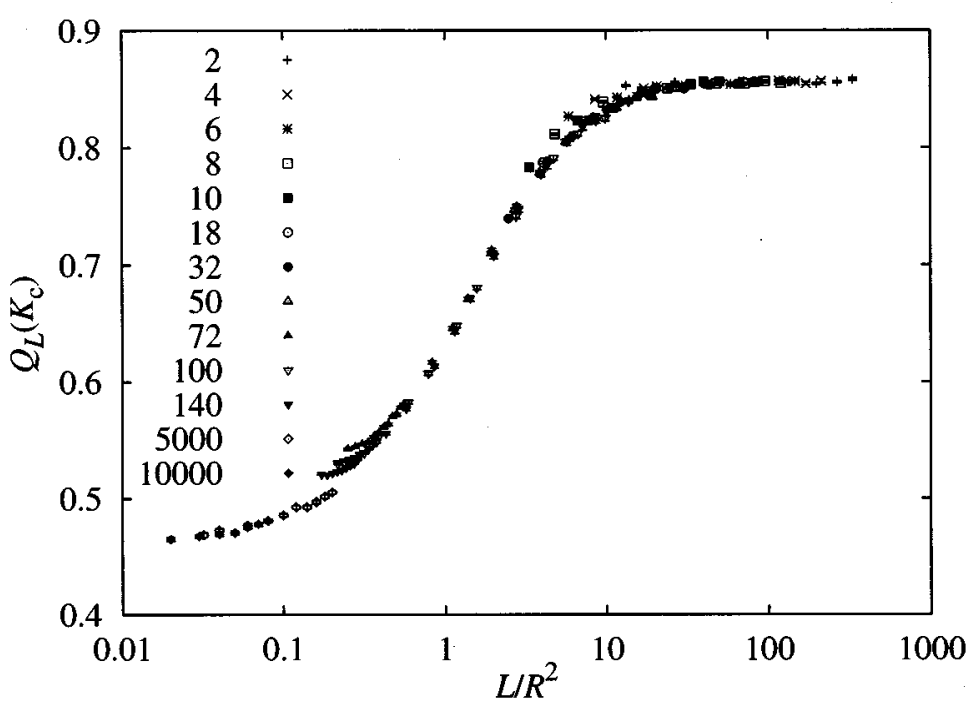

(a)

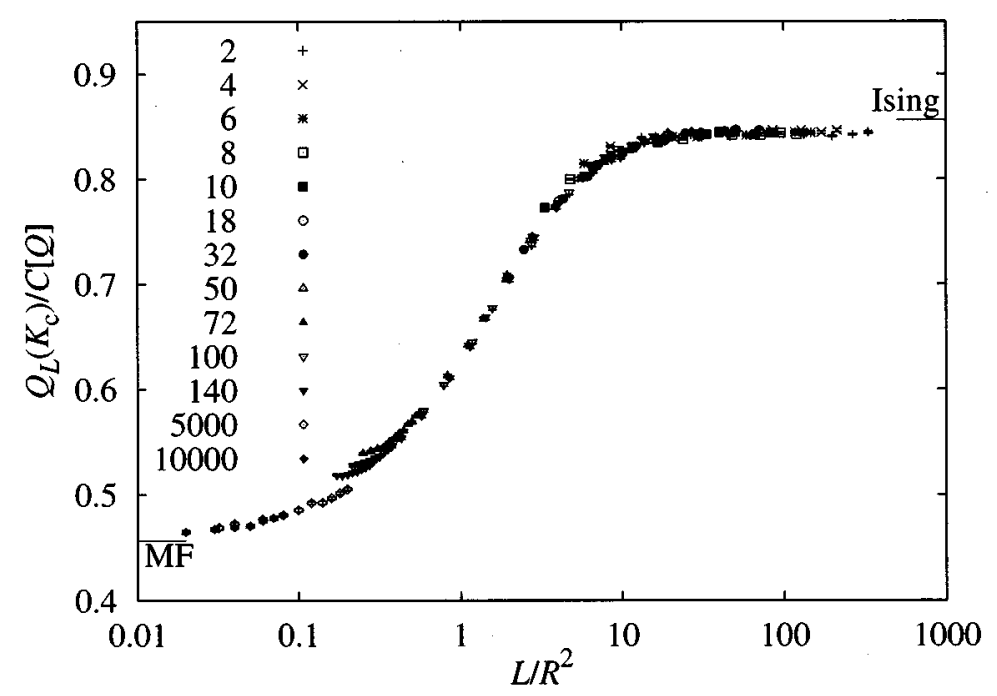

FIG. 6. Finite-size crossover curves for the amplitude ratio $Q \equiv\left\langle m^{2}\right\rangle^{2} /\left\langle m^{4}\right\rangle$. (a) shows the curve without any additional corrections, whereas in (b) a range-dependent correction factor $C[Q]$ [see Eq. (8)] has been divided out. For small values of the crossover variable $L / R^{2}$ the mean-field limit is reproduced and for large values of $L / R^{2}$ the Ising limit is approached. For a further discussion see the text.

(b)

Ref. [10], $Q_{L}\left(K_{c}\right)$ was plotted for a large interaction range $\left(R_{m}^{2}=140\right)$ as a function of the system size. The approach of the Ising value was clearly visible for $L$ large, but for small system sizes $Q$ first decreased toward $Q_{\mathrm{MF}}$ and then started to show strong nonlinear finite-size effects. Evidently, it is a better approach to construct the true crossover curve for $Q\left(K_{c}\right)$ by plotting finite-size data for $Q$ for various ranges versus the crossover variable. This is shown in Fig. 6(a). Several remarks apply to this graph. First, one notes that $L / R^{2}$ is indeed the appropriate crossover variable: A reasonable collapse is obtained for all values of $L$ and $R$. However, some remarkable deviations from this scaling behavior are present, which are most clearly visible in the range 0.2 $<L / R^{2}<0.6$, but also present around $L / R^{2}=10$. Similar effects were already observed in the spin-spin correlation function, but now the effects are much more pronounced because we have employed for the amplitude ratio a linear instead of a logarithmic vertical scale. These deviations are due to nonlinear finite-size corrections, as can be seen clearly by zoom- ing in on the deviations; see Fig. 7. The data points for $R_{m}^{2}$ $=5000$ and $R_{m}^{2}=10000$ may serve as a reference for the location of the "true" crossover curve. One observes that for each of the ranges $R_{m}^{2}=72,100$, and 140 the deviations from this curve increase with decreasing system size, which indeed shows that the effects are caused by finite-size corrections. If the deviations had been caused by, e.g., an inaccurate determination of the critical temperature, the effects would have increased with increasing system size. Unfortunately, it is not easy to separate these corrections from the leading crossover behavior (except graphically), unless the full crossover function is known (which in turn would limit the use of a numerical determination). Of course this problem can be circumvented by determining the crossover at these values for $G$ from systems with a larger system size and a larger interaction range. The deviations around $L / R^{2}$ $=10$ are caused by the same effect, but now for systems with small $R$. Although the amplitude ratio is more sensitive (even if one takes into account the difference in scale) to 


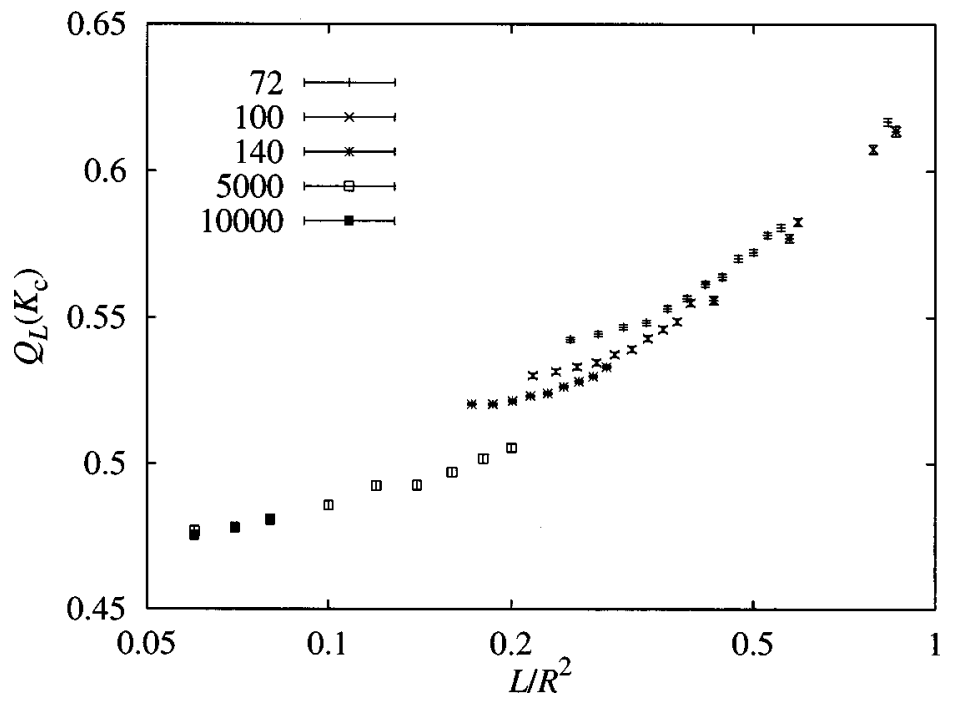

FIG. 7. Detailed view of Fig. 6(a) showing the deviations from the crossover curve for very small system sizes. these finite-size effects than $\left\langle m^{2}\right\rangle=\chi / L^{2}$ and $\left\langle m^{4}\right\rangle$ individually (the curve for the latter is not shown here, but its smoothness is comparable to that of the susceptibility), $Q$ is less sensitive to corrections to the leading range dependence. Indeed, for $\left\langle m^{4}\right\rangle$ these corrections are again of the form [1 $\left.+R^{-2}\left(s_{1}+s_{2} \ln R^{2}\right)+R^{-4} s_{3}\right]$ and $Q$ must thus be divided by

$$
\frac{\left[1+R^{-2}\left(q_{1}+q_{2} \ln R^{2}\right)+R^{-4} q_{3}\right]^{2}}{1+R^{-2}\left(s_{1}+s_{2} \ln R^{2}\right)+R^{-4} s_{3}} .
$$

The coefficients $s_{1}, s_{2}$, and $s_{3}$ have been determined from a least-squares fit to the critical amplitudes of $\left\langle m^{4}\right\rangle$ and $q_{1}, q_{2}$, and $q_{3}$ come from Eq. (7). Figure 8 shows the correction factors for $\left\langle m^{2}\right\rangle,\left\langle m^{4}\right\rangle$, and $Q$. Evidently, the latter factor (8) is much closer to unity than the former two. Figure 6(b) shows $Q_{L}\left(K_{c}\right)$ divided by the correction factor (8), which indeed shows only slightly less scatter than the graph without this correction factor. In particular, the deviations for the larger ranges do not disappear.

\section{THERMAL CROSSOVER SCALING}

\section{A. General considerations}

The finite-size crossover scaling studied in the preceding section is an intrinsic finite-size effect that is not observable in thermodynamic systems. For this reason it is important to study its temperature-dependent counterpart as well. This socalled thermal crossover, which was from a phenomenological scaling point of view already considered in Ref. [16], is of course closely related to finite-size crossover: In finite systems crossover to mean-field-like behavior occurs when the system size has been decreased to the appropriate power of the interaction range (i.e., $L \sim R^{4 /(4-d)}$ or $L \sim R^{2}$ for $d$ $=2$ ), whereas in the temperature-dependent case this crossover occurs when the temperature distance to the critical point is such the correlation length has become of the order of an appropriate power of the interaction range. In the latter case, the precise crossover location is determined by the Ginzburg criterion $t^{(4-d) / 2} R^{d} u^{-1} \approx 1$, where $u$ is the coefficient of the $\phi^{4}$ term in the LGW Hamiltonian. It should be kept in mind that these considerations are valid only within the critical region, i.e., care must be exercised to keep the reduced temperature $t \equiv\left[T-T_{c}(R)\right] / T_{c}(R)$ sufficiently small. When studying thermal crossover in practical simulations one has the additional complication that sufficiently close to $T_{c}$ the correlation length will always be bounded by the finite system size, which is precisely the situation one wants to avoid. So relatively large system sizes are required.

As follows from the Ginzburg criterion, the appropriate scaling variable in two dimensions is $t R^{2}$ and one can there-

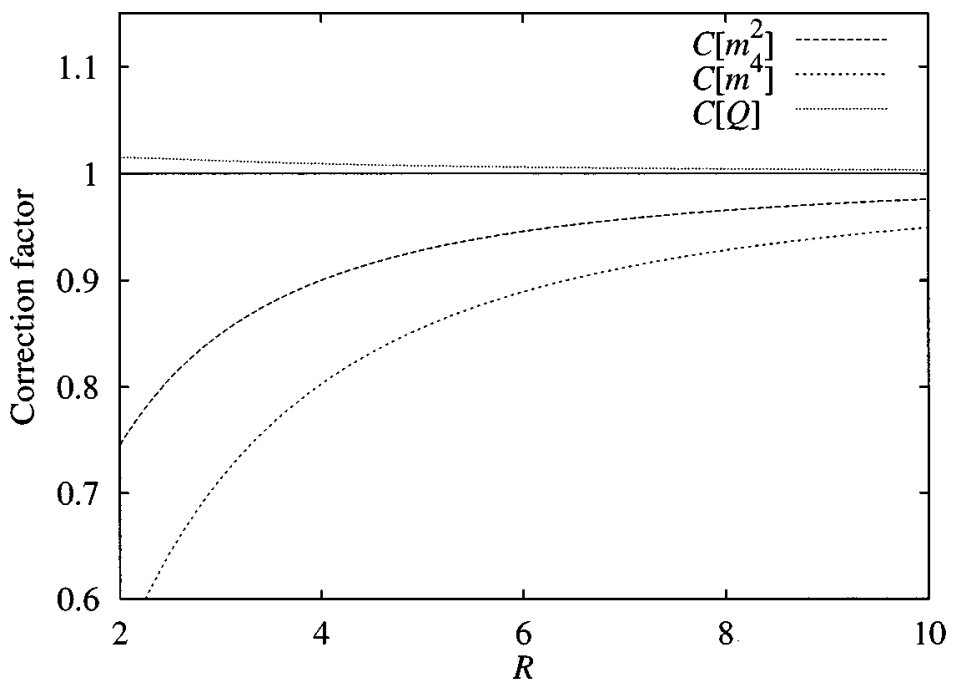

FIG. 8. Range-dependent correction factors $C\left[m^{2}\right]=C[\chi], C\left[m^{4}\right]$, and $C[Q]$ in $\left\langle m^{2}\right\rangle,\left\langle m^{4}\right\rangle$, and $Q$, respectively, as determined by leastsquares fits to the critical amplitudes extracted from the Monte Carlo data. The line at height 1 is drawn for reference. One observes that $C[Q]$ lies very close to, although not exactly at, unity. 
TABLE I. Some properties of the additional ranges used to span the full thermal crossover region. $R_{m}^{2}$ $=5000$ has been included for completeness; it has only been used for the finite-size crossover scaling. The first three columns list the squared range of interaction $R_{m}^{2}$, the corresponding number of neighbors $z$, and the squared effective range of interaction $R^{2}$, respectively. In addition, the critical coupling $K_{c}$ as calculated from Eq. (4) and the mean-field approximation for the critical coupling $K_{c}^{\mathrm{MF}}=1 / z$ are shown.

\begin{tabular}{rrrlc}
\hline \hline \multicolumn{1}{c}{$R_{m}^{2}$} & \multicolumn{1}{c}{$z$} & \multicolumn{1}{c}{$R^{2}$} & \multicolumn{1}{c}{$K_{c}$} & $K_{c}^{\mathrm{MF}}$ \\
\hline 500 & 1580 & $\frac{99449}{395} \approx 251.770$ & $6.379(2) \times 10^{-4}$ & $6.3291139 \times 10^{-4}$ \\
1000 & 3148 & $\frac{394530}{787} \approx 501.309$ & $3.1904(6) \times 10^{-4}$ & $3.1766201 \times 10^{-4}$ \\
4000 & 12580 & $\frac{1259568}{629} \approx 2002.49$ & $7.9594(5) \times 10^{-5}$ & $7.9491256 \times 10^{-5}$ \\
5000 & 15704 & $\frac{9813759}{3926} \approx 2499.68$ & $6.3746(3) \times 10^{-5}$ & $6.3678044 \times 10^{-5}$ \\
10000 & 31416 & $\frac{6545445}{1309} \approx 5000.34$ & $3.18491(9) \times 10^{-5}$ & $3.1830914 \times 10^{-5}$ \\
\hline \hline
\end{tabular}

fore study thermal crossover effects by varying the interaction range as well. This is essential because of the following. For small values of $R, t$ has to be made rather large to cross over to classical critical behavior and it is possible that one leaves the critical region before reaching the classical regime. On the other hand, if one only studies systems with large interaction ranges, $t$ has to be made very small to observe Ising-like critical behavior. However, for such small values of $t$ extremely large system sizes are required to avoid finite-size effects. Therefore, we have constructed, just as in Sec. III, crossover curves from results for various ranges. We have carried out simulations for the interaction ranges studied in Ref. [10] at temperatures further below $T_{c}$ and also generated data for the interaction ranges $R_{m}^{2}=500,1000$, 4000, and 10000 . Table I summarizes some properties of these systems. Simulations have been carried out down to temperatures as low as $T \approx 0.5 T_{c}$. For the order parameter crossover can only be studied in the phase of broken symmetry, but for the susceptibility we have also considered the symmetric $\left(T>T_{c}\right)$ phase. Since in this phase no saturation effects occur, much smaller interaction ranges suffice to span the full crossover region, as we will show below.

\section{B. Absolute magnetization density}

As derived in Refs. [9,10], the absolute magnetization density scales, sufficiently close to the critical point, as $\langle|m|\rangle \propto(-t)^{\beta} R^{(2 d \beta-d) /(4-d)} \quad(t<0)$, which for the twodimensional case yields $\langle|m|\rangle \propto(-t)^{1 / 8} R^{-3 / 4}$. In the meanfield regime, on the other hand, the magnetization density is simply proportional to $(-t)^{1 / 2}$. When plotted as a function of $t R^{2}$, a data collapse for all ranges is now obtained if the magnetization density is multiplied by $R$. Figure 9 (a) shows the corresponding plot. We will discuss the various aspects of this graph in more detail. The overall picture suggests that the data roughly follow the Ising asymptote (slope 1/8) for small values of $t R^{2}$ and then gradually approach the meanfield asymptote (slope 1/2) for large values of $t R^{2}$. Here "small" and "large" refer to the absolute value of $t R^{2}$ and "slope" is generally used for the logarithmic derivative $d \ln \langle|m|\rangle / d \ln |t|$. For very small values of $t R^{2}$ the data start to deviate from the Ising asymptote at an $L$-dependent location and approximately follow (for temperatures closer to $T_{c}$ ) a horizontal line. Here one has entered the finite-size regime, where the correlation length is limited by the system size. This is the case that was studied in Sec. III. The width of this regime depends (for general $d$ ) on both the system size and the interaction range, as can be read off from the universal scaling functions derived in Ref. [10]. Indeed, the temperature-dependent argument of these functions is $t L^{y_{t}} R^{-2\left(2 y_{t}-d\right) /(4-d)} \quad\left(y_{t}=1\right.$ in the 2D Ising universality class) and the width of the finite-size regime is thus proportional to $L^{-y_{t}} R^{2\left(2 y_{\mathrm{t}}-d\right) /(4-d)}=L^{-1}$. Note that the absence of any range dependence is not a general feature and even for the two-dimensional Ising model only true to leading order (cf. Fig. 5 of Ref. [10]). Higher-order terms will entail rangedependent factors that involve (for $d=2$ ) logarithms of $R$. Outside the finite-size regime, the data for each individual range first lie approximately on the Ising asymptote, which has been drawn with an amplitude such that it coincides with the data for $R_{m}^{2}=2$. For the smaller ranges the amplitudes of the asymptotes show a considerable range dependence, whereas for larger ranges the amplitudes converge. Upon further decrease of the temperature (increase of the absolute value of $t$ ) several types of behavior occur: For the smallest range $\left(R_{m}^{2}=2\right)$ the data points still lie on the Ising asymptote. For $R_{m}^{2}=4$ and $R_{m}^{2}=10$ the data leave the Ising asymptote at sufficiently low temperatures and then follow a nearly straight line with a slope that lies between the Ising and the mean-field asymptote. In these cases one has left the critical region without ever reaching the asymptotic mean-field regime. For each range the data for all system sizes coincide, as they should outside the finite-size regime. For $R_{m}^{2}=72$ and $R_{m}^{2}=140$ the mean-field asymptote is approached much more closely. However, if the temperature is decreased further below the critical temperature the data points start to deviate from the asymptote again. This effect is caused by saturation of the magnetization and can be quantitatively described with mean-field theory, as we will show below. Turning to even larger ranges, we see that the data now really reach the asymptote with slope $1 / 2$ and follow it for up to one decade in the crossover variable (for the largest range we have studied) before saturation sets in. Also the exact amplitude $\sqrt{3}$ (see below) of the asymptote is precisely reproduced, which shows again that the critical temperatures of the systems with large interaction ranges have been accurately determined: A deviation would have shifted the graph along the horizontal axis.

We will now first consider the offset of the asymptotes in the Ising regime. Although this effect occurs outside the finite-size regime, we may well hope that the so-called finiterange corrections applied in Sec. III [Eq. (6)] can be used here as well. Indeed, these corrections are part of the univer- 


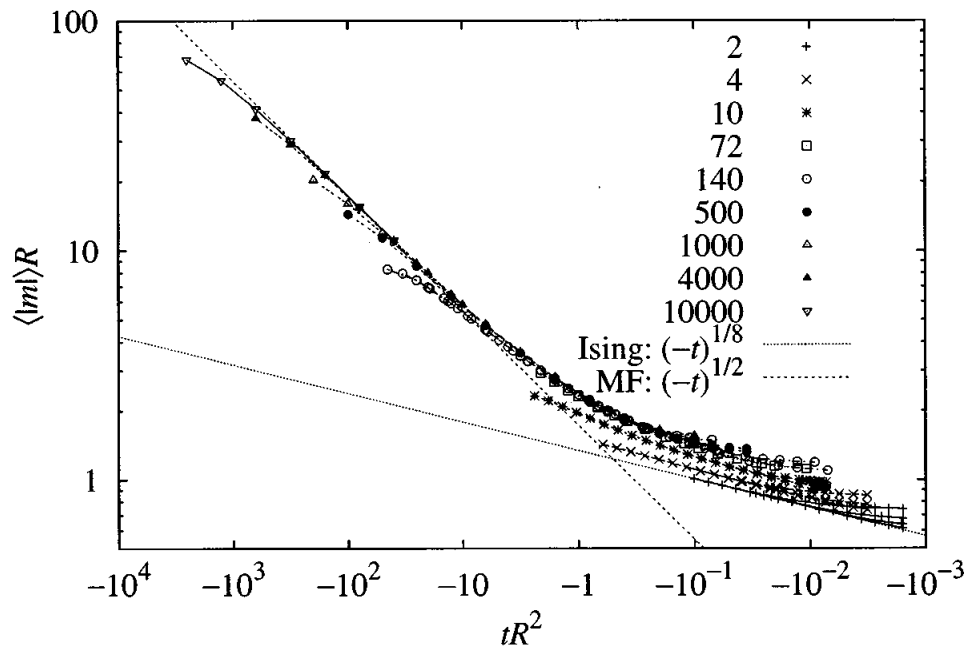

(a)

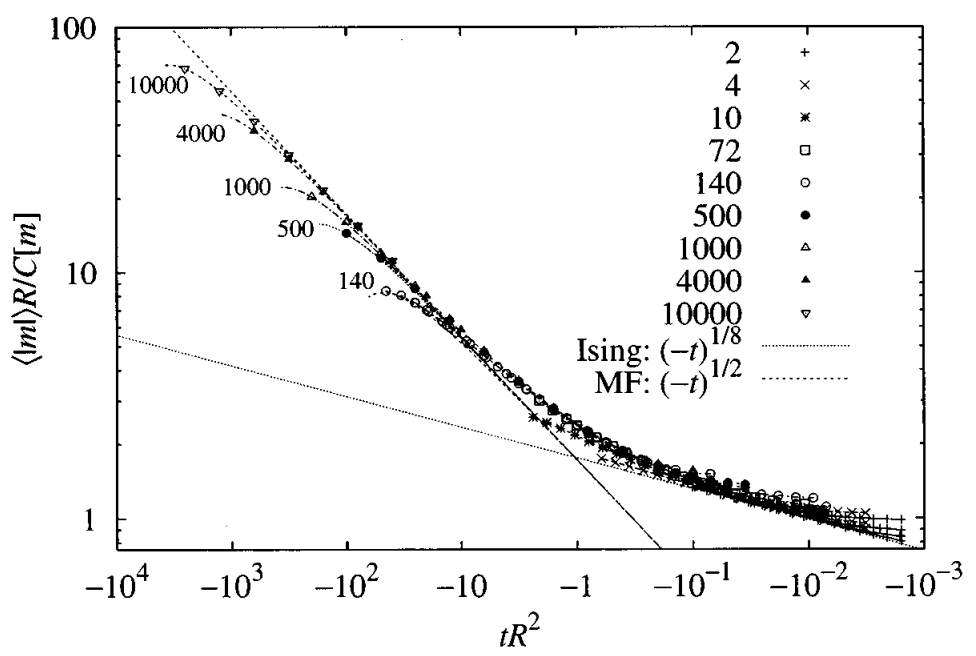

(b)

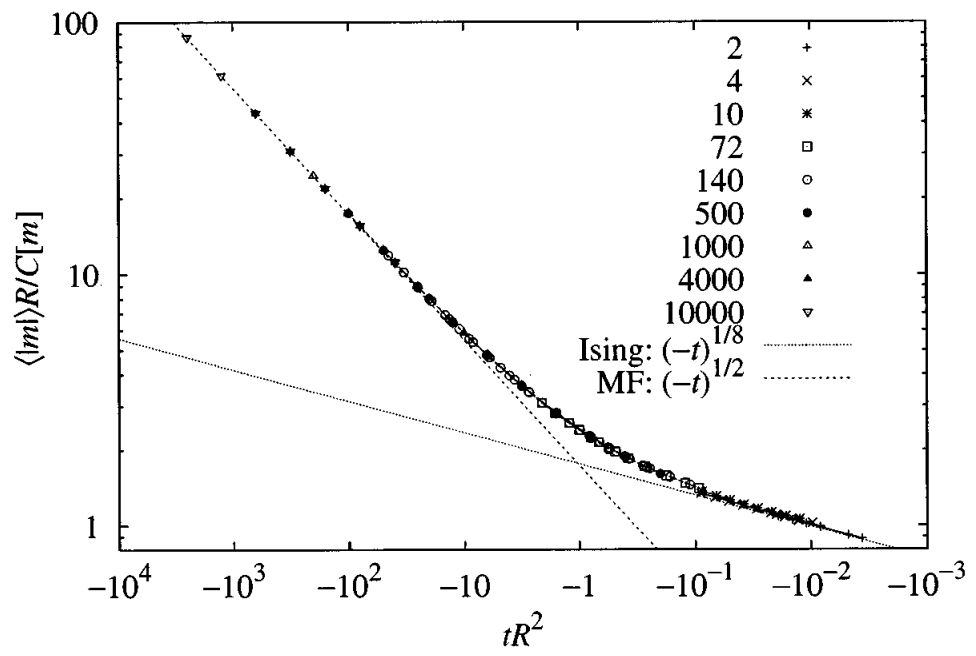

FIG. 9. Thermal crossover for the absolute magnetization density for various ranges and system sizes, where the reduced temperature $t$ is defined as $\left[T-T_{c}(R)\right] / T_{c}(R)$. In (a) no additional correction terms have been used, whereas in (b) the factor $C[m]$ has been divided out. In (c) the data for $R_{m}^{2} \geqslant 72$ have also been corrected for saturation effects and data points in the finite-size regime have been omitted. For an extensive discussion of the various features of these graphs the reader is referred to the text.

(c)

sal scaling functions and although the amplitude $b_{0}$ $=\lim _{R \rightarrow \infty} \lim _{L \rightarrow \infty} R^{3 / 4} L^{1 / 8}\left\langle\left|m_{L}\left(K_{c}\right)\right|\right\rangle$ is a specific limiting value, the range-dependent correction factor does not depend on this limit. Especially the collapse obtained in Fig. 2(b) makes it very tempting to apply a similar correction here. On the other hand, these corrections were calculated in the Ising regime, which we here are gradually leaving. In Fig. 9(b) we show the same data, but now divided by the correction fac- 
tor. Although a perfect collapse is not obtained, the asymptotes lie together much more closly than without this correction.

Also the critical amplitude of the Ising asymptote is known exactly. Indeed, by expanding Onsager's expression for the spontaneous magnetization $[17,18]$

$$
m=\left[1-\frac{1}{\sinh ^{4}\left(2 J / k_{B} T\right)}\right]^{1 / 8}
$$

around the critical point $J / k_{B} T_{c}=\frac{1}{2} \operatorname{arcsinh}(1)$, we obtain for $t<0$

$m=\left[4 \sqrt{2} \operatorname{arcsinh}(1)(-t)+O\left(t^{2}\right)\right]^{1 / 8} \approx 1.22240995(-t)^{1 / 8}$.

For the nearest-neighbor Ising model $R=R_{m}=1$, so the fact that in Fig. 9 along the horizontal axis $t R^{2}$ is used instead of $t$ and along the vertical axis $\langle|m|\rangle R$ instead of $\langle|m|\rangle$ does not affect the amplitude of the asymptote. However, the correction factor $C[m]$ [denoting the factor in square brackets in Eq. (6)] must of course be taken into account. This correction factor describes the deviation of the critical amplitude $d_{0}(R)$ from the leading scaling behavior in terms of a power series in $R^{-2}$ (with coefficients that depend on $\ln R$ ) and it is not a priori clear whether $C[\mathrm{~m}]$ converges for $R=1$. It is certainly unlikely that a single term [the term proportional to $b_{2}$ in Eq. (6) vanishes] describes the deviation very well. No exact result for $d_{0}(R=1)=\lim _{L \rightarrow \infty} m_{L}\left(K_{c}\right) L^{1 / 8}$ is known to us, but from a modest Monte Carlo simulation we found $d_{0}(R=1)=1.0092(4)$. On the other hand, from Eq. (6) with $b_{0}=1.466(2)$ and $b_{1}=-0.305(1)$ we find $d_{0}(R=1)$ $=1.018(4)$, which differs approximately two standard deviations from the numerical result. Recall that $b_{0}$ and $b_{1}$ were obtained from a least-squares fit to the critical finite-size amplitudes for $2 \leqslant R_{m}^{2} \leqslant 140$. Nevertheless, the relative difference lies below the $1 \%$ level, which cannot be distinguished in our graph. Therefore, we have drawn the Ising asymptote with amplitude $[4 \sqrt{2} \operatorname{arcsinh}(1)]^{1 / 8} /\left(1-b_{1}\right)$ in Fig. 9(b) and it indeed turns out to be a precise tangent to the crossover curve.

As mentioned above, also the saturation effects can be described with mean-field theory. Namely, the magnetization follows from the well-known expression $[19,20]$

$$
m=\tanh \left(\frac{T_{c}}{T} m\right) .
$$

Rewriting this as $m=(1+t) \operatorname{arctanh}(m)$ and solving for $m$, one obtains below $T_{c}$ for small $t$

$$
\begin{aligned}
m= & \sqrt{3}(-t)^{1 / 2}-\frac{2}{5} \sqrt{3}(-t)^{3 / 2}-\frac{12}{175} \sqrt{3}(-t)^{5 / 2} \\
& -\frac{2}{125} \sqrt{3}(-t)^{7 / 2}+\frac{166}{67375} \sqrt{3}(-t)^{9 / 2}+O\left((-t)^{11 / 2}\right) .
\end{aligned}
$$

The leading term shows the classical value $\beta=1 / 2$ and the critical amplitude $\sqrt{3}$. To describe the saturation effects in Fig. 9, the first three terms of this series suffice.
Figure 9(b) shows for the five largest ranges $\left(R_{m}^{2}=140,500,1000,4000,10000\right)$ the curves

$$
\begin{aligned}
\langle|m|\rangle R= & \sqrt{3}\left(-t R^{2}\right)^{1 / 2} \\
& \times\left[1-\frac{2}{5 R^{2}}\left(-t R^{2}\right)-\frac{12}{175 R^{4}}\left(-t R^{2}\right)^{2}\right] .
\end{aligned}
$$

For $R_{m}^{2}=140$ this expression does not precisely coincide with the numerical data, but for the remaining values the curves accurately describe the saturation effects. For these cases the interaction ranges are apparently large enough to suppress the critical fluctuations to a large extent. The lowest temperatures shown in the figure are $T / T_{c}=0.52,0.60,0.60$, 0.68 , and 0.50 for $R_{m}^{2}=140,500,1000,4000$, and 10000 , respectively. Saturation effects become visible in Fig. 9 for $t \lesssim-0.15$, i.e., $T / T_{c} \lesssim 0.85$. According to Eq. (12), the magnetization deviates here approximately $5 \%$ from the asymptote. Using Eq. (12), we can perform another operation on the numerical data. Namely, the influence of saturation effects in the mean-field model is described by the ratio of the full series expansion on the right-hand side of Eq. (12) to its first term. As the mean-field expression constitutes an accurate description of the saturation effects for $R_{m}^{2} \geqslant 500$, the factor in square brackets in Eq. (13) will give an accurate description of the relative saturation effects (i.e., the ratio of the saturated magnetization and the crossover curve) down to probably even lower interaction ranges. To illustrate this we have divided the data for $R_{m}^{2} \geqslant 72$ by the corresponding factor. The resulting graph [Fig. 9(c)], in which also the data points in the finite-size regime have been omitted, shows that the data for these large ranges now nicely coincide on one curve, which is the actual crossover curve for the order parameter.

The fact that for different interaction ranges the data (which overlap for considerable intervals of $t R^{2}$ ) coincide on one curve lends strong support to the hypothesis that the crossover curve is universal. Indeed, nonuniversal effects may occur once one has left the critical region. Then microscopic cutoff effects are no longer negligibly small compared to the finite correlation length $\xi$, which implies that the form of the crossover curve depends on the ratio between $\xi$ and the lattice spacing $a$. In our simulations we have not measured the correlation length directly, but we can still make a rough estimate from the data. Namely, at the locations marking the boundaries of the finite-size regime for different interaction ranges and system sizes in Fig. 9, the correlation length is approximately equal to the system size. From the magnetization densities for $R_{m}^{2} \geqslant 72$ we conclude that $\xi$ $\approx 0.5 /(-t)$, independent of the interaction range. The latter conclusion is in agreement with the above-mentioned renormalization prediction that the width of the finite-size regime is to leading order independent of the interaction range. Thus, at a fixed value of the crossover variable $t R^{2}$ the correlation lengths for different ranges have different values. However, the crossover curves coincide at fixed $t R^{2}$ and hence are independent of the ratio $\xi / a$.

Finally, we make some observations concerning the size of the crossover region. It is clear that it takes between two 


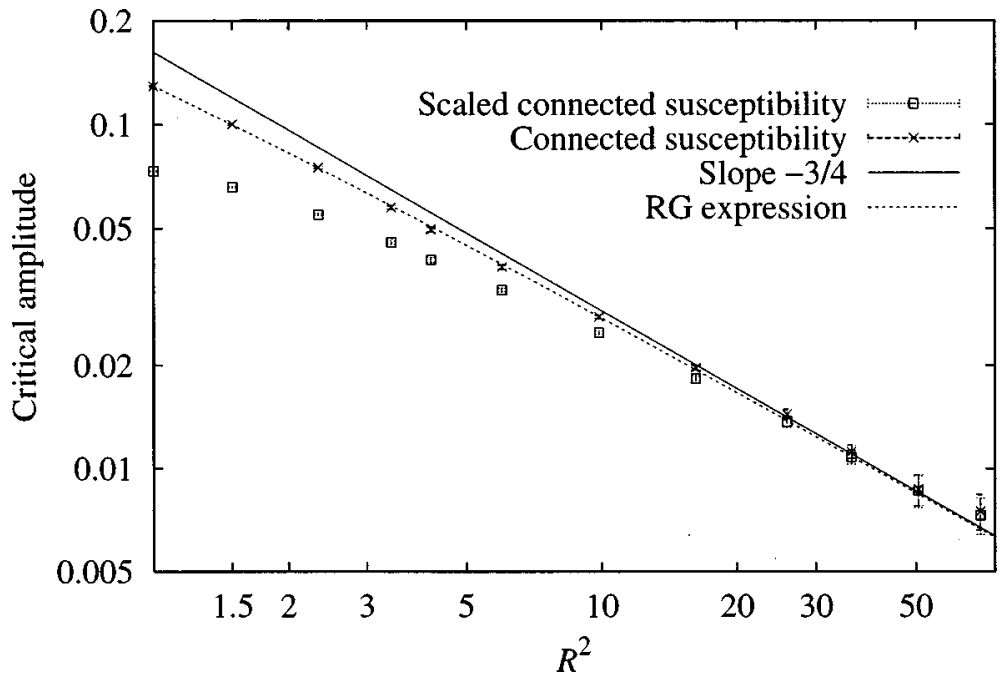

FIG. 10. Critical amplitude for the connected susceptibility $\tilde{\chi}=L^{d}\left(\left\langle m^{2}\right\rangle-\langle|m|\rangle^{2}\right) / k_{B} T$ as extracted from the thermodynamic limit of $L^{-7 / 4} \widetilde{\chi}_{L}\left(K_{c}\right)$. The dashed curve indicates the renormalization prediction fitted to the numerical data. Also the critical amplitude of the scaled susceptibility $k_{B} T_{c} \tilde{\chi}$ is shown, which for small ranges deviates considerably stronger from the asymptotic behavior. and three decades in the crossover variable to cross over from Ising-like to classical critical behavior. Thus, unless one studies systems with a rather large interaction range, one has to go to such a large temperature distance from $T_{c}$ to sufficiently decrease the correlation length compared to the interaction range that one has already left the critical region before observing classical critical behavior. The center of the crossover region lies in the neighborhood of $\left|t R^{2}\right|=1$, consistent with a value for $u$ of order unity.

\section{Magnetic susceptibility}

Unlike the order parameter, the magnetic susceptibility displays crossover upon approaching the critical point either from below or from above. We will discuss these two situations separately. In the ordered phase, $T \leqslant T_{c}$, we approximate the magnetic susceptibility by the so-called connected susceptibility

$$
\tilde{\chi}=L^{d} \frac{\left\langle m^{2}\right\rangle-\langle|m|\rangle^{2}}{k_{B} T} .
$$

In the two-dimensional Ising model with interaction range $R$ this quantity will, close to the critical point, diverge as $(-t)^{-7 / 4} R^{-3 / 2}$. Further below $T_{c}$ it will cross over to classical critical behavior, where $\tilde{\chi} \propto(-t)^{-1}$. In a graph showing results for various ranges as a function of the crossover variable $t R^{2}$ a data collapse is obtained for $\tilde{\chi} / R^{2}$. However, just as for previous crossover curves, the data for small $R$ will display an offset because of corrections to the leading $R^{-3 / 2}$ dependence. To determine these deviations we first study the critical amplitude of the connected susceptibility, which was not considered in Ref. [10]; see Fig. 10. The statistical uncertainty of this amplitude is notably larger than for $\langle|m|\rangle$ and $\left\langle m^{2}\right\rangle$ (cf., e.g., Fig. 3), but one can still observe that the asymptotic regime is reached. In this figure we have also plotted the critical amplitude of the so-called scaled susceptibility $k_{B} T \tilde{\chi}$, which was studied in, e.g., Ref. [9]. Evidently, the latter amplitude shows a much stronger deviation from the leading range dependence, due to the fact that also $T_{c}(R)$ deviates from $T_{c}^{\mathrm{MF}}$ (Fig. 1). Thus, although both amplitudes have the same asymptotic behavior for large interaction ranges, it is much more difficult to extract this behavior from medium-range results for $k_{B} T \tilde{\chi}$ than from the corresponding results for $\tilde{\chi}$. This may partially explain the difficulties experienced in Ref. [9]. The deviations have been fitted to a correction factor of the form $\left[1+R^{-2}\left(v_{1}+v_{2} \ln R^{2}\right)\right]$, which we abbreviate as $C[\widetilde{\chi}]$. Indeed, the fact that the finite-range corrections for $\tilde{\chi}$ are so small allowed us to neglect them altogether in Ref. [11], where only logarithmic scales have been employed.

In Fig. 11(a) we show the connected susceptibility, appropriately scaled with $R$ and divided by the correction factor $C[\tilde{\chi}]$, as a function of the crossover variable. Just as for the magnetization density, deviations from the crossover curve are present even after the finite-range corrections have been applied. These effects are either caused by finite-size effects (close to $T_{c}$ ) or by systems that leave the critical region. In the latter case, saturation effects start to come into play. The finite-size effects are clearly recognizable in the rightmost part of the graph, where the curves start to follow horizontal lines. Once the temperature has been sufficiently decreased, the graphs start following an asymptote with slope $-7 / 4$, on which the data for various ranges quite accurately collapse. The amplitude of this asymptote is simply related to the exactly known amplitude $A^{-}=0.025537 \ldots$ [21,22] of the reduced susceptibility $\chi_{0}$. This reduced susceptibility is defined as $\chi_{0} \equiv k_{B} T \widetilde{\chi} / \mu^{2}$, where $\mu$ denotes the magnetic moment of a spin. This magnetic moment has been divided out implicitly in our calculations as well. However, we should keep in mind that we have expressed all temperatures in terms of the mean-field critical temperature, i.e., $T_{c}$ $=1 / z K_{c}$, where $z$ is the coordination number. For the nearest-neighbor model this yields an additional factor 4 and we thus expect a critical amplitude $2 \operatorname{arcsinh}(1) A^{-}$. In addition, we have to take into account the finite-range correction factor that has been divided out. The question whether this factor is applicable for $R=1$ has already been discussed in Sec. IV B [below Eq. (10)]. Here the difference between the deviation from the leading scaling behavior as predicted by $C[\widetilde{\chi}]$ and the numerical result is approximately $3 \%$, whereas the smallest differences that can be discerned on the logarithmic scale of Fig. 11(a) are of the order of 5\%. The asymptote with the above-mentioned amplitude divided by $C[\widetilde{\chi}]$ in- 


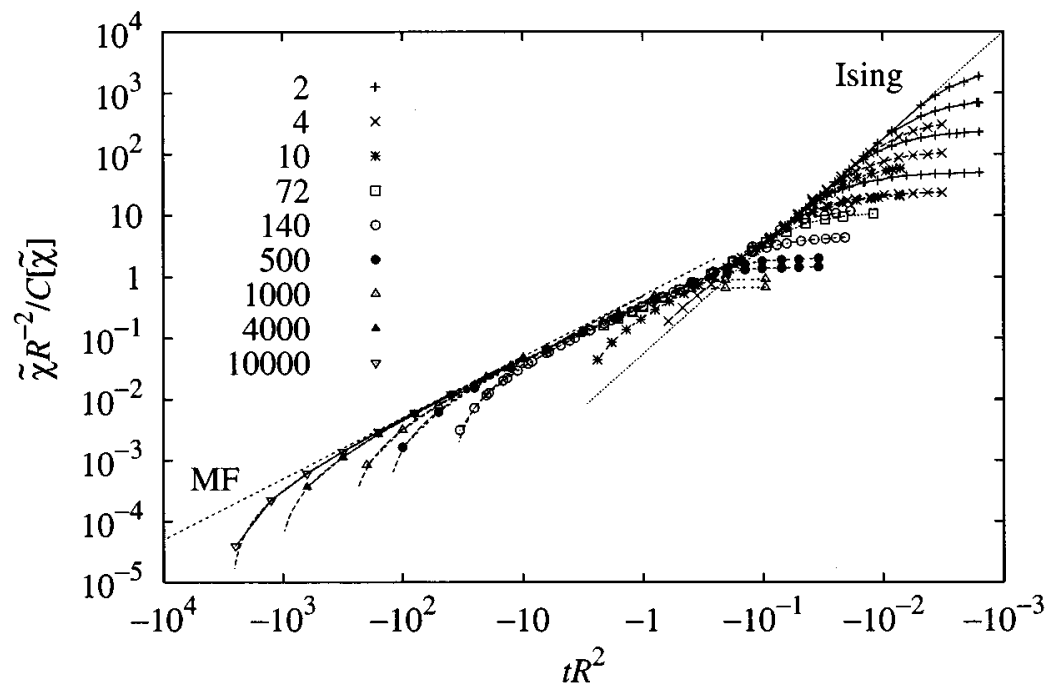

(a)

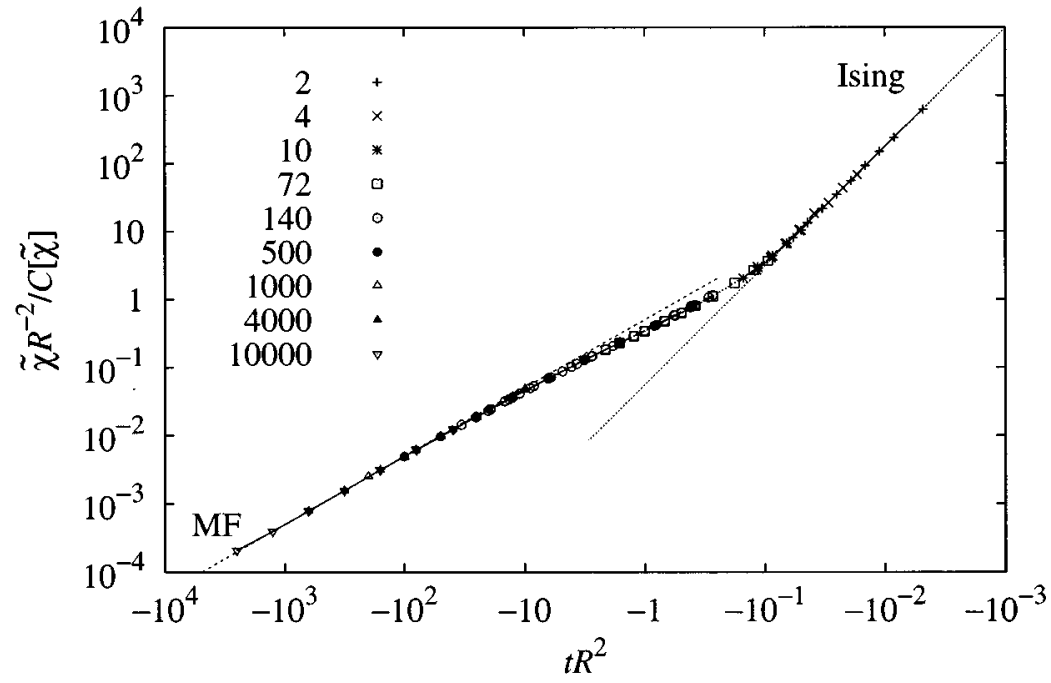

FIG. 11. Thermal crossover for the connected susceptibility $\tilde{\chi}$ for various ranges and system sizes. A finite-range correction factor $C[\tilde{\chi}]$ has been divided out. (b) has also been corrected for saturation effects for $R_{m}^{2} \geqslant 72$ and data points in the finite-size regime have been omitted. For a discussion see the text.

(b)

deed lies tangential to the crossover curve, confirming our data. As the temperature is further decreased, the data for systems with small interaction ranges start to follow a line with a slope between that of the Ising and the mean-field asymptotes. This effect is caused by the fact that these systems have left the critical region. For sufficiently large interaction ranges, however, the curves coincide and have a slope that gradually decreases (in the absolute sense). Although the crossover curve at first varies more rapidly than for the magnetization density, it subsequently only slowly approaches the classical regime and the overall size of the crossover region is again between two and three decades. Remarkably, the slope of the crossover curve passes even through the mean-field value -1 before settling at this value for sufficiently low temperatures. In other words, the derivative of the connected susceptibility appears to change nonmonotonically from its asymptotic Ising value $-7 / 4$ to its classical value -1 . Several explanations may be considered for this behavior. Either it is an intrinsic effect of the crossover function or it might be explained from the fact that $\tilde{\chi}$ is the difference between $\left\langle m^{2}\right\rangle$ and $\langle|m|\rangle^{2}$, which each separately are described by a monotonically varying curve.
The saturation effects can, just as for the magnetization density, for large ranges be described with mean-field theory. In a mean-field model the magnetic susceptibility is given by

$$
\chi=\frac{1-m^{2}}{t+m^{2}} .
$$

Using Eq. (12) we find for $T<T_{c}$

$$
\begin{aligned}
\chi= & \frac{1}{-2 t}-\frac{9}{10}+\frac{18}{175}(-t)+\frac{18}{175}(-t)^{2}+\frac{6714}{67375}(-t)^{3} \\
& +O\left((-t)^{4}\right),
\end{aligned}
$$

which exhibits the classical value for the susceptibility exponent $\gamma_{\mathrm{MF}}=1$ and the critical amplitude $\frac{1}{2}$. Figure 11(a) shows the asymptote with this amplitude and one can observe that the crossover curve approaches this asymptote from below around $t R^{2}=-1$. Also the mean-field curves (16) are shown for $R_{m}^{2}=140,500,1000,4000$, and 10000 and they accurately describe the numerical data. Thus we have used the ratio between the series expansion (16) and the asymptotic 


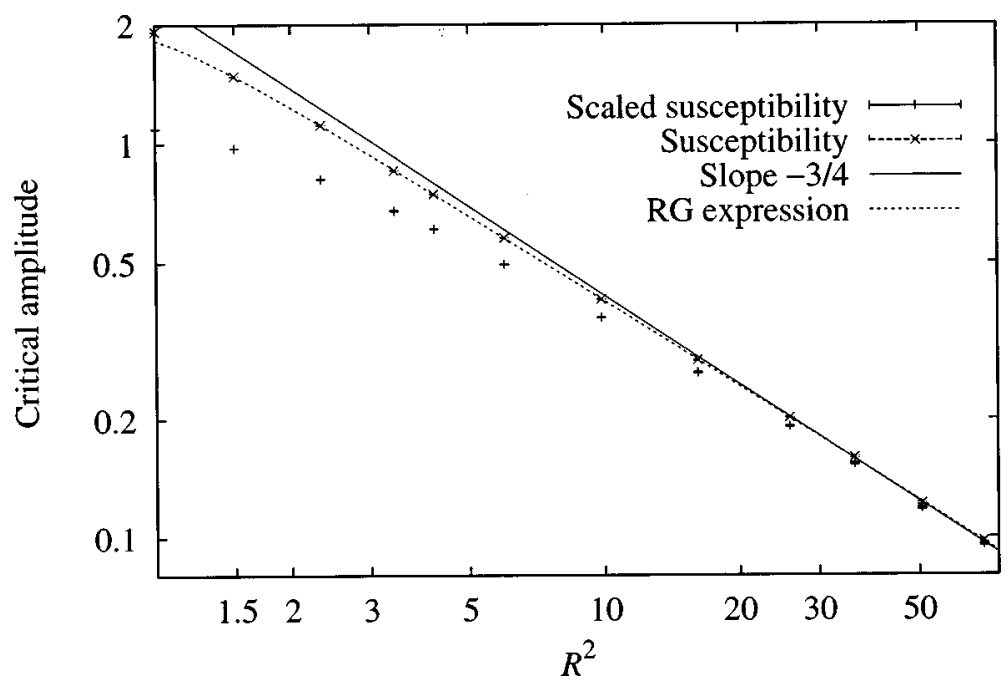

FIG. 12. Critical amplitude for the susceptibility $\chi^{\prime}=L^{d}\left\langle m^{2}\right\rangle / k_{B} T$ as extracted from the thermodynamic limit of $L^{-7 / 4} \chi_{L}^{\prime}\left(K_{c}\right)$. The dashed curve indicates the renormalization prediction fitted to the numerical data. Also the critical amplitude of the scaled susceptibility $k_{B} T_{c} \chi^{\prime}$ is shown, which for small ranges deviates considerably stronger from the asymptotic behavior.

behavior $1 /(-2 t)$ to remove the saturation effects in Fig. 11(a). The resulting graph is shown in Fig. 11(b), in which also the data points in the finite-size regime have been omitted in order to obtain a clear crossover curve. The nonmonotonic variation of the slope of this curve is clearly visible.

In the disordered (symmetric) phase, we encounter a different situation. The susceptibility is now given by $\chi^{\prime}$ $\equiv L^{d}\left\langle m^{2}\right\rangle / k_{B} T$. This is identical to the expression we have used for the finite-size crossover scaling, except that the temperature-dependent factor has been omitted in Sec. III C. Figure 12 shows the critical finite-size amplitudes of both $\chi^{\prime}$ and $\chi=L^{d}\left\langle m^{2}\right\rangle$ as a function of the interaction range. We have fitted an expression of the form (7) to the data for $R_{m}^{2}$ $\geqslant 2$. This expression describes the data well, except for the data point at $R_{m}^{2}=1$, where the deviation is approximately $10 \%$. Just as for the connected susceptibility, the finite-range corrections to the critical amplitude of $\chi^{\prime}$ are much smaller than for $\chi$. In fact, they are so small that they can be completely omitted in the thermal crossover scaling, as illustrated in Fig. 13. This graph shows $\chi^{\prime} / R^{2}$ as a function of the crossover variable $t R^{2}$ for various interaction ranges and system sizes. Outside the finite-size regime, the data follow the Ising asymptote with slope $-7 / 4$. The exactly known amplitude $2 \operatorname{arcsinh}(1) A^{+}$, where $A^{+}=0.96258 \ldots[21,22]$, of this asymptote is accurately reproduced by the numerical data. For larger temperatures, the curves gradually approach an asymptote with the mean-field slope -1 . However, some care has to be exercised when interpreting this behavior. Above $T_{c}$, no saturation of the order parameter occurs and the system smoothly passes over to regular (noncritical) behavior. In this high-temperature region the susceptibility decreases proportionally to $1 / T$. For small interaction ranges it is this behavior that one observes in the graph. Only for larger interaction ranges one actually observes classical critical behavior. The latter systems indeed reproduce the meanfield critical amplitude, which is equal to 1 [as follows from Eq. (15) with $m=0]$. Note that, due to the absence of saturation effects, interaction ranges up to $R_{m}^{2}=1000$ are amply sufficient to observe the full crossover region.

\section{EFFECTIVE EXPONENTS}

In several papers (see, e.g., Refs. [5,2]) the slopes of the crossover functions are described by so-called effective exponents. These exponents can be defined as $\beta_{\mathrm{eff}} \equiv d \ln \langle m\rangle / d \ln |t|=t d \ln \langle m\rangle / d t \quad$ and $\quad \gamma_{\mathrm{eff}} \equiv-d \ln \chi / d \ln |t|=$ $-t d \ln \chi / d t$. In fact, this concept has been familiar from the analysis of experimental data for a long time [23], but only a limited amount of theoretical work has addressed these issues. Of course, these exponents change from their Ising val-

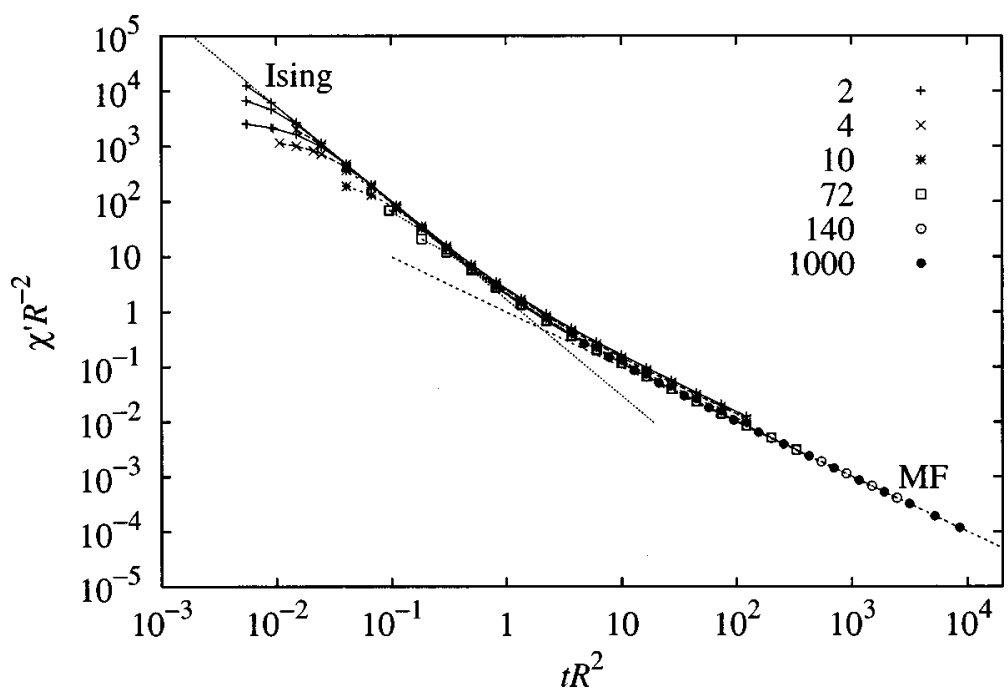

FIG. 13. Thermal crossover for the susceptibility $\chi^{\prime}$ in the symmetric phase for various ranges and system sizes. No finite-range corrections have been applied. For a discussion see the text. 


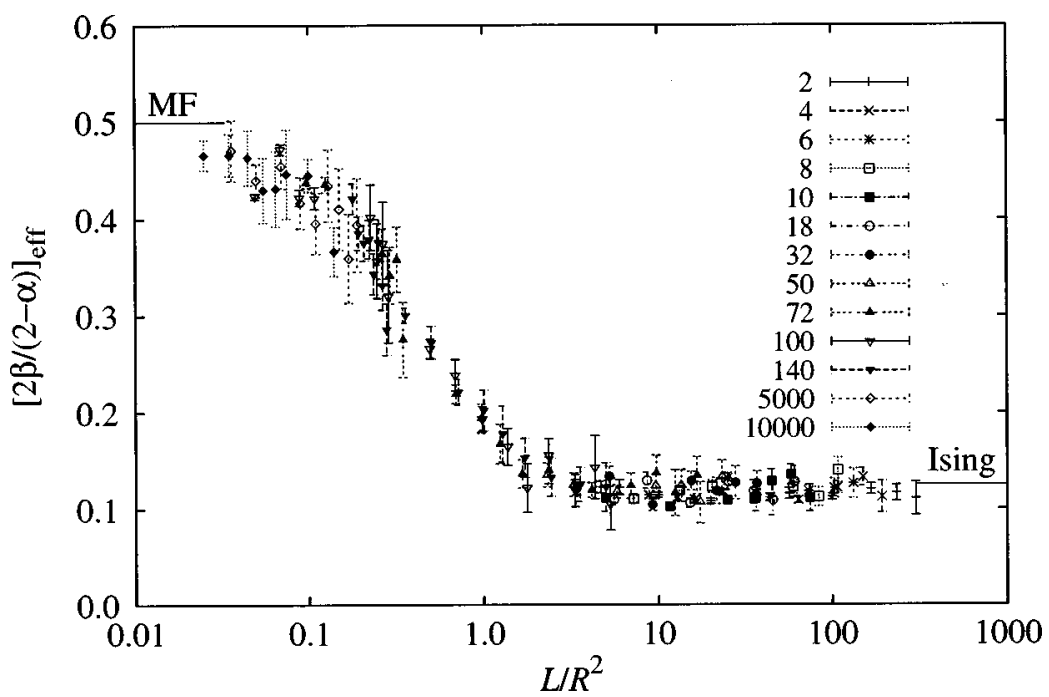

FIG. 14. Effective exponent $[2 \beta /(2-\alpha)]_{\mathrm{eff}}$ as obtained from the finite-size crossover curve for $\langle|m|\rangle \sqrt{L}$. ues to the classical values in the crossover region. However, the precise variation in the crossover region is unclarified and partially subject to debate. Although these exponents can be read off from the form of the crossover curves presented in Sec. IV, we consider it worthwhile to present separate graphs displaying $\beta_{\text {eff }}, \gamma_{\text {eff }}^{-}$, and $\gamma_{\mathrm{eff}}^{+}$, where the superscripts denote the cases $t<0$ and $t>0$, respectively. The additional advantage of these exponents is that they follow from data with the same range and hence are not affected by any rangedependent correction factors.

As the graph in Fig. 2(b) is particularly smooth, it is tempting to consider its derivative as well. As derived in Ref. [10], $\langle|m|\rangle \sqrt{L} \propto\left(L / R^{2}\right)^{y_{h}-3 / 2}$. This relation also holds in the mean-field regime, provided that one replaces the magnetic exponent $y_{h}$ by its starred counterpart $y_{h}^{*} \equiv 3 d / 4=3 / 2$. The asterisk indicates that the exponent is modified due to the dangerous-irrelevant-variable mechanism, as explained in, e.g., Ref. [24]. Thus, while we can rewrite the abovementioned relation in the Ising regime in terms of conventional critical exponents as $\langle|m|\rangle \sqrt{L} \propto\left(L / R^{2}\right)^{-\beta / \nu+1 / 2}$, this is not possible in the mean-field regime since $\nu_{\mathrm{MF}}$ is not affected by the dangerous-irrelevant-variable mechanism. As an alternative we employ the specific-heat exponent $\alpha$ : $\langle|m|\rangle \sqrt{L} \propto\left(L / R^{2}\right)^{-2 \beta /(2-\alpha)+1 / 2}$. The fact that the latter rela- tion holds in the mean-field regime while the former does not is a direct manifestation of the violation of hyperscaling. Thus we define $[2 \beta /(2-\alpha)]_{\mathrm{eff}} \equiv \frac{1}{2}-d \ln (\langle|m|\rangle \sqrt{L}) / d \ln (L /$ $R^{2}$ ). This quantity is shown as a function of $L / R^{2}$ in Fig. 14 . Although the error bars are considerable, the crossover from the Ising value $1 / 8$ (for large values of $L / R^{2}$ ) to the classical value $1 / 2$ (for small values of $L / R^{2}$ ) is clearly visible.

Turning to thermal crossover, we display in Figs. 15, 16, and 17 the exponents $\beta_{\text {eff }}, \gamma_{\text {eff }}^{-}$, and $\gamma_{\text {eff }}^{+}$, respectively, as defined above. The effective magnetization exponent $\beta_{\text {eff }}$ increases monotonically from its Ising value $1 / 8$ to the classical value $1 / 2$. In particular, the data for different interaction ranges roughly fall onto the same curve, which supports the hypothesis that the crossover curve is universal. However, one observes that for systems with relatively small interaction ranges the effective exponent does not follow this curve. This effect, caused by saturation of the order parameter, can clearly lead to misleading results in experiments. In Fig. 16 the nonmonotonic variation of $\gamma_{\text {eff }}^{-}$between $7 / 4$ and 1 is clearly visible. This may be considered as a manifestation of what Fisher [5] calls an "underswing." The occurrence of such a nonmonotonic crossover has been predicted by various renormalization calculations for the crossover from Ising

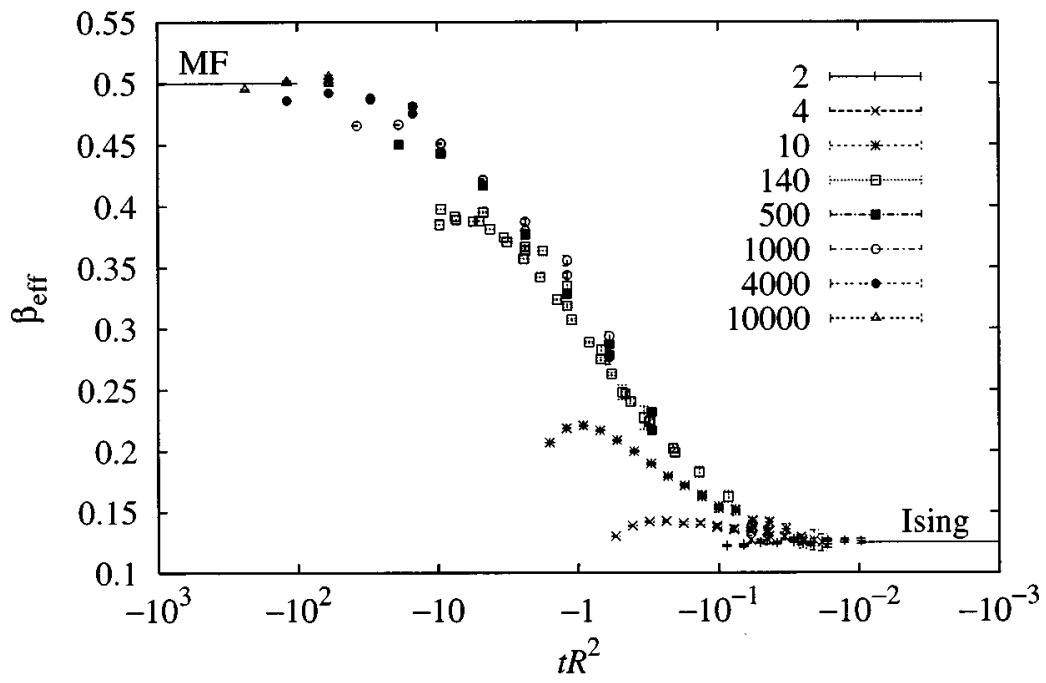

FIG. 15. Effective magnetization exponent $\beta_{\text {eff }}$ describing the logarithmic derivative of the crossover function for the magnetization density. 


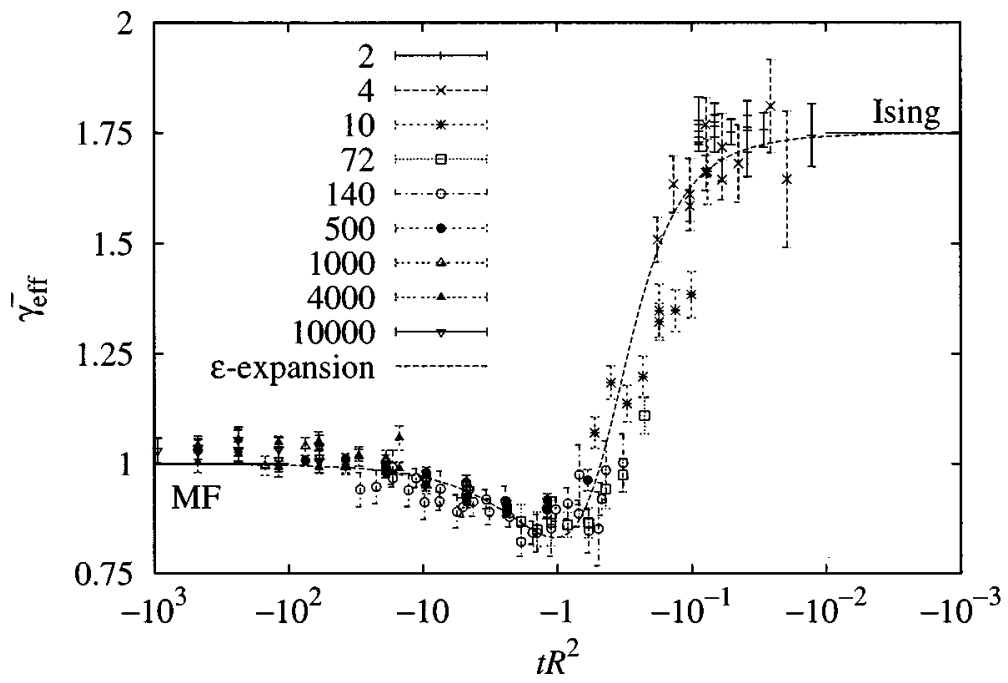

FIG. 16. Effective susceptibility exponent $\gamma_{\text {eff }}^{-}$ describing the logarithmic derivative of the crossover function for the connected susceptibility. The results on the left-hand side lie somewhat above the mean-field exponent due to saturation effects. to $X Y$ and Heisenberg critical behavior above $T_{c}$; see, e.g., Refs. [25-27] and references therein. Furthermore, an exponent $\gamma_{\text {eff }}=0.88(3)$ has been measured in the symmetric phase in micellar solutions [28]. Fisher [5] has suggested that an effective susceptibility exponent that takes a value $\gamma_{\text {eff }}$ $<1$ in the crossover region might be a general feature of crossover from 3D Ising to classical critical behavior and noted that concrete calculations yielding such an effective exponent would be valuable. In Ref. [5], a first-order $\varepsilon$ expansion is quoted for the exponent crossover function

$$
\gamma_{\mathrm{eff}}=1+\left(\gamma_{I}-\gamma_{\mathrm{MF}}\right) E[\ln (|t / G|)],
$$

where $G$ is the crossover temperature or Ginzburg number and

$$
E(\ln y)=1 /\left(1+y^{\varepsilon / 2}\right)
$$

In our case, $t / G$ is directly proportional to the crossover variable $t R^{2}$. To describe the experimental results from Ref. [28], Fisher used an extension of Eq. (18),

$$
E(\ln y)=\left(1+p y^{\varepsilon / 2}\right) /\left[1+(p+1) y^{\varepsilon / 2}+q y^{\varepsilon}\right] .
$$

Even though one may not expect such an expansion to converge for $d=2$, we have drawn expression (17) in Fig. 16, where we have taken the function $E(\ln y)$ from Eq. (19), set $\varepsilon=2$, and adjusted $p$ and $q$ such that the curve constituted a reasonable description of the data. Clearly, no conclusions should be drawn from this curve, especially because Eq. (19) has been proposed for the symmetric phase. In addition, for $d=2$ the exponent $\varepsilon / 2$ is a very poor approximation for the exponent $\theta \equiv-y_{i} / y_{t}=2$, which is actually expected to appear in the function $E(\ln y)$. As follows from Fig. 17, the behavior above $T_{c}$ is completely different. Here we have used expression (17) with Eq. (18) to describe the data. Except for a shift along the horizontal axis (a proportionality constant in the Ginzburg number), no adjustable parameter is present and it is surprising how well the data agree with the theoretical prediction. It would be interesting to calculate the amplitude of the first Wegner correction as a function of $R$. However, even with the present techniques this would, for the large values of $R$, require prohibitively large system sizes (to avoid finite-size effects) and thus has not been attempted.

Sometimes experiments have yielded effective exponents in disagreement with the known [29] universality classes, but still satisfying the scaling relations, such as $\gamma_{\mathrm{eff}}+2 \beta_{\mathrm{eff}}=2$ $-\alpha_{\text {eff }}$. Here $\alpha_{\text {eff }}$ denotes the effective exponent of the specific heat, which in our case is expected to be always (close to) zero, as both the classical and the 2D Ising value of $\alpha$ are equal to zero. This is also confirmed by the close resem-

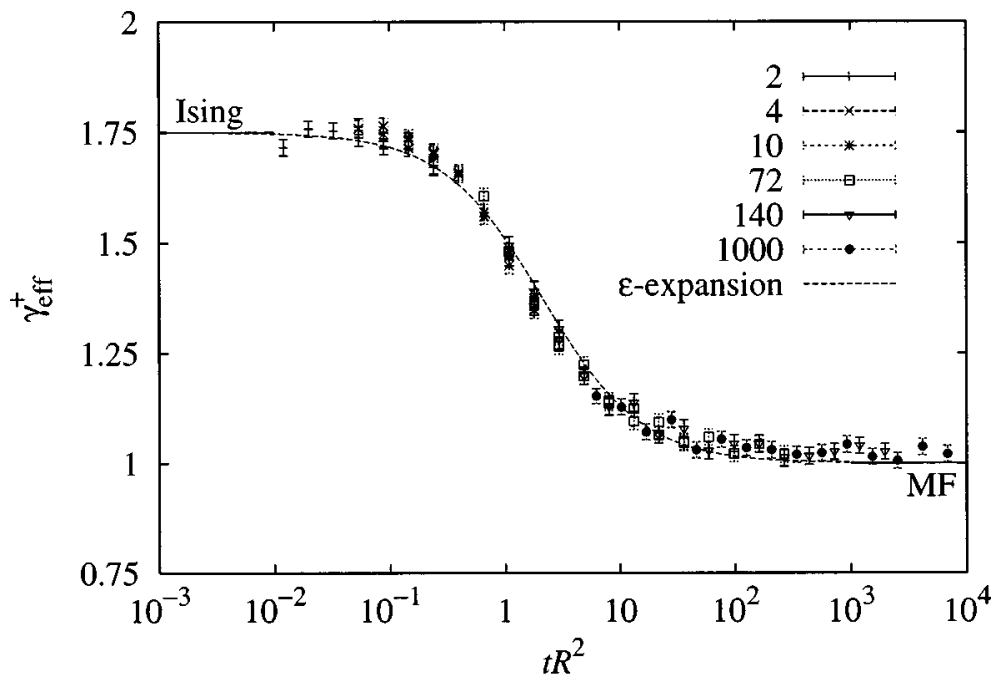

FIG. 17. Effective susceptibility exponent $\gamma_{\mathrm{eff}}^{+}$ describing the logarithmic derivative of the crossover function for the susceptibility above $T_{c}$. 
blance between Figs. 14 and 15. Thus it is interesting to note that this scaling relation is strongly violated in the present case: From Figs. 15 and 16 we can estimate that $\gamma_{\text {eff }}$ $+2 \beta_{\text {eff }}$ reaches a minimum of approximately 1.4 at $t R^{2} \approx$ -1 .

\section{CONCLUSIONS}

In this paper we have presented numerical results for scaling functions describing the crossover from Ising-like to classical critical behavior in two-dimensional systems. While the general concepts describing this crossover have been developed many years ago, only a limited amount of progress has been made for a long time. In the present paper it is demonstrated that one can obtain accurate quantitative information on crossover scaling from computer simulations. The full crossover region was covered for both finite-size crossover and thermal crossover above and below $T_{c}$. A data collapse has been obtained for all system sizes and interaction ranges, which supports the hypothesis that these crossover functions are universal. Deviations from this curve are present, but can be understood from finite-size and saturation effects. The results are in agreement with the previously derived renormalization scenario for these systems.

Working in two dimensions offers the advantage that the exponents and the critical amplitudes are known exactly. More importantly, critical fluctuations are very large in two dimensions, which leads to critical behavior that strongly differs from classical behavior and hence to a clearly visible crossover between the two universality classes. We have shown that the magnetization density is described by a smooth crossover curve. The effective exponent, defined as the logarithmic derivative of this curve, increases monotonically from the Ising value to the classical value in two or three decades in the reduced temperature. On the other hand, the effective exponent for the susceptibility has a logarithmic derivative that varies monotonically above the Curie temperature and nonmonotonically below it. The occurrence of nonmonotonic behavior in the symmetric phase has been inferred from renormalization-group calculations in three dimensions and found long-standing interest. An extension of the present study to $d=3$ is therefore highly desirable and has been planned for the near future.

\section{ACKNOWLEDGMENTS}

E.L. and H.B. acknowledge the kind hospitality of the condensed-matter theory group of the Johannes GutenbergUniversität Mainz, where part of this work has been completed. We are grateful to the Höchstleistungsrechenzentrum
Jülich for access to a Cray-T3E computer, on which part of the computations have been carried out.

\section{APPENDIX: EXACT CALCULATION OF SOME FINITE-SIZE EFFECTS IN A MEAN-FIELD SYSTEM}

In Ref. [14] the universal amplitude ratio $Q$ has been calculated for a system in which all spins interact equally strongly, including the leading finite-size correction. It was shown that the relevant integrals can be expressed in terms of a quantity $I_{k}$, which we here generalize to odd powers of the magnetization density,

$$
I_{k} \equiv \int_{-\infty}^{\infty} d m|m|^{k} \exp \left(-\frac{1}{12} N m^{4}\right)=\left(\frac{12}{N}\right)^{(k+1) / 4} \frac{1}{2} \Gamma\left(\frac{k+1}{4}\right) \text {, }
$$

where $N$ denotes the number of spins. In a similar fashion we can also calculate other finite-size effects to leading order in $N$. Expanding the terms in the partition function and replacing the sum over all possible states by an integral, we find for the absolute magnetization density

$$
\begin{aligned}
\langle|m|\rangle= & \frac{1}{Z} \int_{-\infty}^{\infty} d m|m| \exp \left(-\frac{1}{12} N m^{4}\right)\left[1-\frac{1}{30} N m^{6}+\frac{1}{2} m^{2}\right. \\
& \left.+O\left(N m^{8}, m^{4}\right)\right]
\end{aligned}
$$

in which $Z$ denotes the partition function [except for a prefactor that has been divided out; cf. Eq. (31) in Ref. [14]]. Elementary algebra then leads to

$$
\langle|m|\rangle=12^{1 / 4} \frac{\Gamma\left(\frac{1}{2}\right)}{\Gamma\left(\frac{1}{4}\right)} \frac{1}{N^{1 / 4}}+O\left(\frac{1}{N^{3 / 4}}\right)
$$

which for $d=2$ yields expression (5). Along the same lines one finds

$$
\left\langle m^{2}\right\rangle=\sqrt{12} \frac{\Gamma\left(\frac{3}{4}\right)}{\Gamma\left(\frac{1}{4}\right)} \frac{1}{N^{1 / 2}}+O\left(\frac{1}{N}\right),
$$

i.e., the susceptibility diverges as $\sqrt{N}$, and

$$
\left\langle m^{4}\right\rangle=12 \frac{\Gamma\left(\frac{5}{4}\right)}{\Gamma\left(\frac{1}{4}\right)} \frac{1}{N}+O\left(\frac{1}{N^{3 / 2}}\right)=\frac{3}{N}+O\left(\frac{1}{N^{3 / 2}}\right) .
$$

[1] V. L. Ginzburg, Fiz. Tverd. Tela 2, 2031 (1960) [Sov. Phys. Solid State 2, 1824 (1960)].

[2] M. A. Anisimov, A. A. Povodyrev, V. D. Kulikov, and J. V. Sengers, Phys. Rev. Lett. 75, 3146 (1995).

[3] C. Bagnuls and C. Bervillier, Phys. Rev. Lett. 76, 4094 (1996).

[4] M. A. Anisimov, A. A. Povodyrev, V. D. Kulikov, and J. V. Sengers, Phys. Rev. Lett. 76, 4095 (1996).
[5] M. E. Fisher, Phys. Rev. Lett. 57, 1911 (1986).

[6] M. A. Anisimov, S. B. Kiselev, J. V. Sengers, and S. Tang, Physica A 188, 487 (1992).

[7] M. E. Fisher and B. P. Lee, Phys. Rev. Lett. 77, 3561 (1996).

[8] H.-P. Deutsch and K. Binder, J. Phys. II 3, 1049 (1993).

[9] K. K. Mon and K. Binder, Phys. Rev. E 48, 2498 (1993). 
[10] E. Luijten, H. W. J. Blöte, and K. Binder, Phys. Rev. E 54, 4626 (1996).

[11] E. Luijten, H. W. J. Blöte, and K. Binder, Phys. Rev. Lett. 79, 561 (1997).

[12] K. Binder and H.-P. Deutsch, Europhys. Lett. 18, 667 (1992).

[13] E. Brézin and J. Zinn-Justin, Nucl. Phys. B: Field Theory Stat. Syst. 257FS, 867 (1985).

[14] E. Luijten and H. W. J. Blöte, Int. J. Mod. Phys. C 6, 359 (1995).

[15] G. Kamieniarz and H. W. J. Blöte, J. Phys. A 26, 201 (1993).

[16] E. Riedel and F. Wegner, Z. Phys. 225, 195 (1969).

[17] L. Onsager, Nuovo Cimento Suppl. 6, 261 (1949)

[18] B. M. McCoy and T. T. Wu, The Two-Dimensional Ising Model (Harvard University Press, Cambridge, MA, 1973).

[19] W. L. Bragg and E. J. Williams, Proc. R. Soc. London, Ser. A 145, 699 (1934).
[20] R. J. Baxter, Exactly Solved Models in Statistical Mechanics (Academic, London, 1982).

[21] E. Barouch, B. M. McCoy, and T. T. Wu, Phys. Rev. Lett. 31, 1409 (1973).

[22] T. T. Wu, B. M. McCoy, C. A. Tracy, and E. Barouch, Phys. Rev. B 13, 316 (1976).

[23] J. S. Kouvel and M. E. Fisher, Phys. Rev. 136, A1626 (1964).

[24] E. Luijten and H. W. J. Blöte, Phys. Rev. Lett. 76, 1557 (1996); 76, 3662(E) (1996).

[25] D. R. Nelson and E. Domany, Phys. Rev. B 13, 236 (1976).

[26] D. J. Amit and Y. Y. Goldschmidt, Ann. Phys. (N.Y.) 114, 356 (1978).

[27] P. Seglar and M. E. Fisher, J. Phys. C 13, 6613 (1980).

[28] M. Corti and V. Degiorgio, Phys. Rev. Lett. 55, 2005 (1985).

[29] M. E. Fisher, Rev. Mod. Phys. 46, 597 (1974). 